\title{
Making Pointer Analysis More Precise by Unleashing the Power of Selective Context Sensitivity
}

TIAN TAN, Nanjing University, China

YUE LI*, Nanjing University, China

XIAOXING MA, Nanjing University, China

CHANG XU, Nanjing University, China

YANNIS SMARAGDAKIS, University of Athens, Greece

Traditional context-sensitive pointer analysis is hard to scale for large and complex Java programs. To address this issue, a series of selective context-sensitivity approaches have been proposed and exhibit promising results. In this work, we move one step further towards producing highly-precise pointer analyses for hard-to-analyze Java programs by presenting the Unity-Relay framework, which takes selective context sensitivity to the next level. Briefly, Unity-Relay is a one-two punch: given a set of different selective context-sensitivity approaches, say $S=S_{1}, \ldots, S_{n}$, Unity-Relay first provides a mechanism (called Unity) to combine and maximize the precision of all components of $S$. When Unity fails to scale, Unity-Relay offers a scheme (called Relay) to pass and accumulate the precision from one approach $S_{i}$ in $S$ to the next, $S_{i+1}$, leading to an analysis that is more precise than all approaches in $S$.

As a proof-of-concept, we instantiate Unity-Relay into a tool called BATON and extensively evaluate it on a set of hard-to-analyze Java programs, using general precision metrics and popular clients. Compared with the state of the art, BATON achieves the best precision for all metrics and clients for all evaluated programs. The difference in precision is often dramatic-up to $71 \%$ of alias pairs reported by previously-best algorithms are found to be spurious and eliminated.

\section{CCS Concepts: • Theory of computation $\rightarrow$ Program analysis.}

Additional Key Words and Phrases: Pointer Analysis, Alias Analysis, Context Sensitivity, Java

\section{ACM Reference Format:}

Tian Tan, Yue Li, Xiaoxing Ma, Chang Xu, and Yannis Smaragdakis. 2021. Making Pointer Analysis More Precise by Unleashing the Power of Selective Context Sensitivity. Proc. ACM Program. Lang. 5, OOPSLA, Article 147 (October 2021), 27 pages. https://doi.org/10.1145/3485524

\section{INTRODUCTION}

Pointer analysis is important for an array of real-world applications such as bug detection [Chandra et al. 2009; Naik et al. 2006], security analysis [Arzt et al. 2014; Livshits and Lam 2005], program verification [Fink et al. 2008; Pradel et al. 2012] and program understanding [Li et al. 2016; Sridharan

${ }^{*}$ Corresponding author

Authors' addresses: Tian Tan, State Key Laboratory for Novel Software Technology, Nanjing University, China, tiantan@ nju.edu.cn; Yue Li, State Key Laboratory for Novel Software Technology, Nanjing University, China, yueli@nju.edu.cn; Xiaoxing Ma, State Key Laboratory for Novel Software Technology, Nanjing University, China, xxm@nju.edu.cn; Chang Xu, State Key Laboratory for Novel Software Technology, Nanjing University, China, changxu@nju.edu.cn; Yannis Smaragdakis, Department of Informatics and Telecommunications, University of Athens, Greece, yannis@smaragd.org.

This work is licensed under a Creative Commons Attribution 4.0 International License.

(C) 2021 Copyright held by the owner/author(s).

2475-1421/2021/10-ART147

https://doi.org/10.1145/3485524

Proc. ACM Program. Lang., Vol. 5, No. OOPSLA, Article 147. Publication date: October 2021. 
et al. 2007]. A more precise pointer analysis is always favored by its clients as it implies, e.g., fewer false alarms of bugs and vulnerabilities, more accurate code navigation, or potential for optimization.

Context sensitivity has been demonstrated to be a major scheme for improving precision of Java pointer analysis [Fegade and Wimmer 2020; Feng et al. 2015; Kanvar and Khedker 2016; Lhoták and Hendren 2008; Lhoták and Hendren 2006; Milanova et al. 2002, 2005; Tan et al. 2017; Thakur and Nandivada 2019, 2020; Thiessen and Lhoták 2017; Whaley and Lam 2004; Xu and Rountev 2008]. With years of development, traditional context sensitivity performs well for small/medium programs, e.g., 2obj (object-sensitive pointer analysis with context length being two) can analyze most DaCapo benchmarks rapidly and precisely [Kastrinis and Smaragdakis 2013; Smaragdakis et al. 2011]; however, it is hard for it to scale to large and complex Java programs with good precision [Smaragdakis et al. 2014]. (An analysis not scaling means that it blows up in complexity and does not terminate even under very high time limits.)

To address this problem, a series of research work [Hassanshahi et al. 2017; Jeon et al. 2019; Jeong et al. 2017; Li et al. 2018a,b, 2020; Lu and Xue 2019; Minseok Jeon and Oh 2020; Oh et al. 2014, 2015; Smaragdakis et al. 2014; Wei and Ryder 2015] propose to apply context sensitivity selectively in the sense that contexts no longer apply to all methods, while context elements (e.g., objects, types, call-sites) and context lengths may vary for different selected methods. Other, non-selected, methods are treated context-insensitively, in order to enable good scalability.

Conversely, to gain better precision, selective context-sensitivity approaches usually have to sacrifice efficiency by allowing more methods to be analyzed context-sensitively, sometimes with longer context lengths or more precise but heavier context elements. In other words, these analyses need to make careful precision and efficiency trade-offs, where one more step towards precision may put the analysis at the risk of unscalability [ $\mathrm{Li}$ et al. 2020]. As a result, seeking further precision improvement becomes hard, as limited efficiency margins are left to play with. Under such constraints, if the approach for more precise pointer analysis is not designed well, it may introduce significant overhead with minor or no precision improvement (e.g., by selecting for sensitive treatment many precision-useless methods). More commonly, the analysis will choose to scale, but will sacrifice precision (e.g., by selecting very few methods to treat context sensitively).

Problem. For many real-world applications where points-to or alias information is required, such as certain bug detectors, security analyzers, etc., good precision is much favored, even if the price is to run the analysis for a long time (e.g., several hours) [Christakis and Bird 2016]. Thus, the challenge we seek to address is to achieve, given a long but reasonable time allowance, precise pointer analysis results for large and complex Java programs, for which traditional context-sensitive analyses fail to scale, and selective context-sensitive analyses scale but with limited precision.

Method. We introduce a general (meta-)framework called Unity-Relay to produce highly precise pointer analyses for hard-to-analyze Java programs, by systematically exploiting and utilizing any selective context sensitivity technique. Unity-Relay is a one-two punch, with precision as its first priority:

- Unity-Relay takes as inputs a program P and a set of different selective context-sensitivity approaches, $S$;

- it first provides a method (called Unity) to unify all approaches in $S$ (playing the role of a meta-heuristic), maximizing precision by applying the most stringent context-sensitivity selection made by any approach in $S$;

- if Unity fails to scale, the Relay method is employed. It divides the scalability burden of Uni ty into different passes (each pass is a run of context-sensitive pointer analysis guided by an approach in $S$ ), with the precision achieved by one pass being transmitted to and 
accumulated in the next pass: in each pass, the points-to results retrieved from the previous pass are used to on-the-fly filter intermediate points-to results, keeping inferred points-to sets small and, thus, enhancing scalability.

Given any selective context-sensitive pointer analysis $A$ (guided by an approach in $S$ ), if $A$ is sound and scalable for $\mathrm{P}$, no matter how precise $A$ is, Unity-Relay can be expected to produce a sound and scalable analysis with a precision that is guaranteed to be at least as good as $A$ 's, in the worst case. (Experimental results show that the precision is always better.) This property also implies that even when new (possibly special-purpose) selective context-sensitivity insights are developed in the future, Unity-Relay will still be able to leverage them to produce a more precise analysis in practice.

Results. As a proof-of-concept, we instantiate the Unity-Relay framework in a tool called BATON. We extensively evaluate BATON on a set of hard-to-analyze Java programs (including the toughest programs evaluated in the past literature of selective context-sensitive pointer analysis for Java), using three general precision metrics and four popular clients (also the most complete set of precision metrics/clients used in recent literature). Compared to the state of the art, experimental results show that BATON is able to improve precision significantly, and achieves the best precision for all metrics and clients for all evaluated programs. To the best of our knowledge, this is the first time that this level of analysis precision has been attained for these hard-to-analyze programs. Moreover, because of Unity-Relay's nature as a general meta-framework, we expect it to see more future instantiations and to unleash the power of more selective context-sensitivity approaches, to produce more precise pointer analyses.

In summary, this work makes the following contributions:

- We present Unity-Relay, a simple and practical framework to produce precise pointer analyses for hard-to-analyze Java programs, by unleashing the power of selective context sensitivity (Section 3).

- We formulate the Unity-Relay framework, prove its soundness and precision guarantees, and discuss its scalability (Section 4).

- We present BATON, a tool instantiated from the Unity-Relay framework, which will be released as an open-source tool (Section 5).

- We conduct extensive experiments by comparing BATON with the state of the art, to demonstrate its effectiveness in the real world (the experimental results can be obtained using the accompanying artifact [Tan et al. 2021]) (Section 6). BATON substantially improves on the precision of previous algorithms in the literature-e.g., eliminating over $33 \%$ of alias pairs (soundly determined to be spurious) on average, and up to $71 \%$, compared to the best past contender evaluated.

\section{BACKGROUND}

We assume that the reader is familiar with the high-level concepts of pointer analysis, as introduced in several surveys [Smaragdakis and Balatsouras 2015; Sridharan et al. 2013]. This section describes some necessary background knowledge about context sensitivity for whole-program pointer analysis for Java.

Traditional Context Sensitivity. A method is analyzed context-sensitively means that the static abstraction of different dynamic instantiations of variables and heap objects within the method will be treated separately during analysis under different abstract entities called contexts. Accordingly, spurious object flows will be reduced, thus making the analysis more precise. Given a method $\mathrm{m}$, each of its contexts typically consists of a list of consecutive context elements in the form of 
$[c 1, c 2, \ldots]$ to model different run-time conditions. For example, for call-site sensitivity, $c 1$ is the call site of $\mathrm{m}$, and $\mathrm{c} 2$ is the call site of the method containing $\mathrm{c} 1$, etc. However, for efficiency, only the most recent $l$ context elements are kept ( $l$ is also called the context length) and $l$ is usually limited to a small number for whole-program analysis in practice [Kastrinis and Smaragdakis 2013; Smaragdakis et al. 2011]. There are three mainstream context-sensitivity variants for Java pointer analysis: call-site sensitivity, object sensitivity, and type sensitivity. Henceforth, we use 3obj to denote object sensitivity with context length being 3 . Longer context indicates more precision: more spurious data flows can be possibly eliminated. Thus, 3obj is more precise than 2obj. For different kinds of context elements with the same length, past work has demonstrated that object sensitivity typically outperforms call-site sensitivity, in terms of both precision and efficiency [Milanova et al. 2005; Smaragdakis et al. 2011], and is more precise but also more expensive than type sensitivity [Smaragdakis et al. 2011; Tan et al. 2017]. Traditionally, context-sensitive pointer analysis uniformly applies context sensitivity to every method in a program to maintain high precision. However, this approach often does not work for large and complicated programs as such treatment is too costly to scale [Li et al. 2018b; Smaragdakis et al. 2014].

Selective Context Sensitivity. Selective context sensitivity proposes to apply context sensitivity selectively, only for precision-useful/non-scalability-threat methods. The analysis precision of such methods will help improve the overall program analysis precision, while not incurring so much cost as to make the analysis hard to scale. The remaining methods are analyzed context-insensitively so that the space and time cost originally incurred for these methods (in traditional context sensitivity) is saved, leaving room to produce precise and scalable analyses even for hard-to-analyze programs. However, it is challenging to accurately determine which methods are precision-useful but not scalability-threat in general. To deal with this problem, in the past years, various selective context-sensitivity approaches have been presented, based on different insights and policies. For example, the target methods could be selected according to expert experience [WALA 2018], program patterns [Li et al. 2018a, 2020], abstracted memory capacity [Li et al. 2018b], parameterized heuristics [Hassanshahi et al. 2017; Smaragdakis et al. 2014], or machine learning approaches [Jeon et al. 2018; Jeong et al. 2017]. In addition, in selective context sensitivity, some approaches apply the same context sensitivity to the selected methods [Hassanshahi et al. 2017; Li et al. 2018a, 2020; Smaragdakis et al. 2014], while in other approaches context elements and lengths may vary for different subsets of the selected methods [Jeon et al. 2019; Jeong et al. 2017; Li et al. 2018b]. We refer the readers to Section 7 for more details.

\section{THE UNITY-RELAY FRAMEWORK, INFORMALLY}

We first give an overview of the Unity-Relay framework (Section 3.1), and then explain the key ideas of Unity (Section 3.2) and Relay (Section 3.3), respectively.

\subsection{Overview}

Figure 1 shows a pictorial overview of the Unity-Relay framework: given a program P and a set of selective context-sensitive (C.S.) approach/strategy components, $S$, the framework will yield highly precise pointer analysis results for $P$.

Unity-Relay is a one-two punch. It first calls its Unity component. Taking as input the results obtained by running each approach in $S$ for $\mathrm{P}$, the context-sensitivity selector of Unity (C.S. Selector in Figure 1) unifies them and makes new configuration about which portion of $P$ should be analyzed by what context-sensitivity variants (i.e., what are the context elements and lengths) to maximize the precision, according to the unity principle, as introduced in Section 3.2. Intuitively, the Unity 


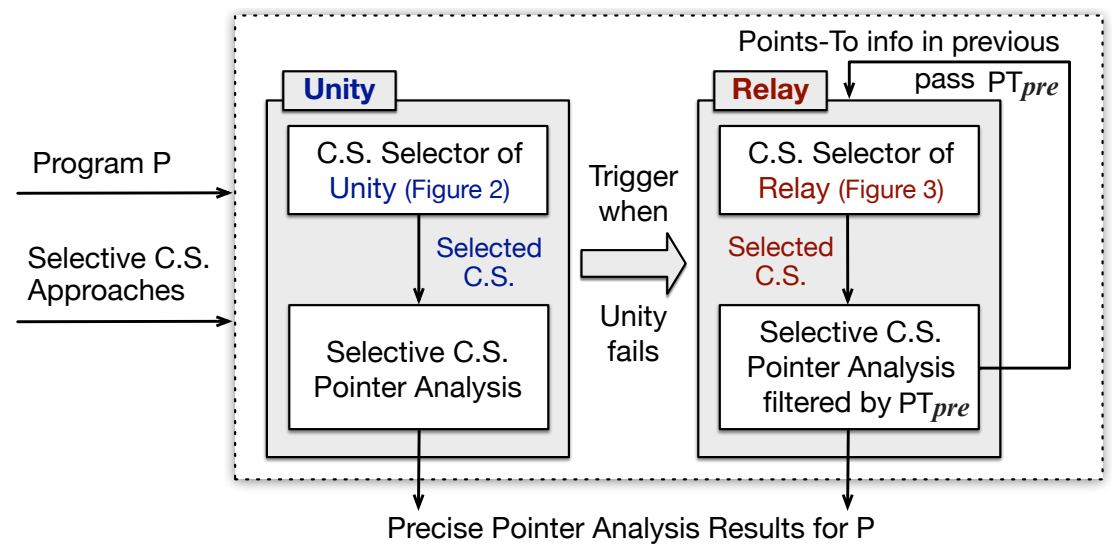

Fig. 1. Overview of the Unity-Relay Framework.

principle maintains for each program element the most precise context that any component contextsensitivity approach would use. Then the new selected context-sensitivity information (Selected C.S. in Figure 1) will guide the selective context-sensitive pointer analysis to analyze $P$, and its results will be the output of Unity-Relay if the analysis is able to finish running within the time limit; otherwise (i.e., if Unity is too expensive to scale), Unity-Relay will trigger its Relay component to analyze $P$.

Generally, given $n$ approaches in $S$, Relay will run the selective context-sensitive pointer analysis $n$ times (i.e., we have $n$ passes in Relay). In each pass, as shown in Figure 1, the selective contextsensitive pointer analysis is guided by the context-sensitivity information (Selected C.S.) output from the C.S. selector of Relay according to the relay principle as illustrated in Section 3.3. Between successive passes, the precision of the earlier pass will be transmitted to and accumulated in the next pass. This is achieved by soundly filtering the pointer analysis results in the current pass by using the points-to information (points-to sets of variables) from the previous pass ( $\mathrm{PT}_{\text {pre }}$ in Figure 1). As a result, in Relay, the scalability burden is shared in each analysis run (i.e., each pass): a component strategy is not burdened by others, but instead each strategy that completes helps both the precision and the scalability of the rest. Although overall precision is not guaranteed to reach that of Unity, precision improvements are reaped in a stable and accumulative way.

\subsection{Unity}

Recall that every selective context-sensitive analysis represents a precision and efficiency trade-off, where precision bumps up against the limits of analysis scalability. In other words, for past selective context-sensitive analyses, the degree of precision (typically: the proportion of the program to be analyzed context-sensitively) is chosen to be approximately at a point where further improving it would render the analysis non-scalable. According to previous work, treating context-sensitively even a small set of methods may significantly hinder scalability, for the "wrong" choice of methods [Li et al. 2020]. Now it seems that we are trapped: if a small step towards precision may hit the scalability wall (in the precision and efficiency balance made by each selective context-sensitivity approach), how can we reap a further noticeable precision improvement in a general, policy-agnostic meta-framework? To escape from the trap, in Unity, we propose to take a big step forward towards higher precision, by allowing many more methods to be analyzed under context sensitivity, with 


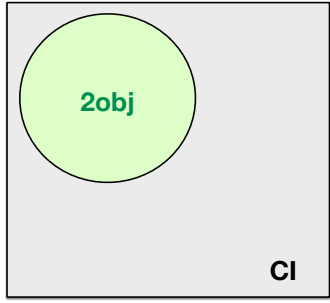

S1

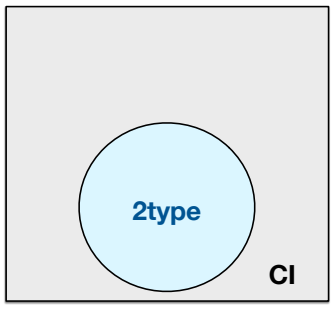

S2

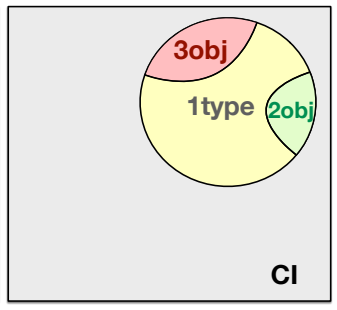

S3

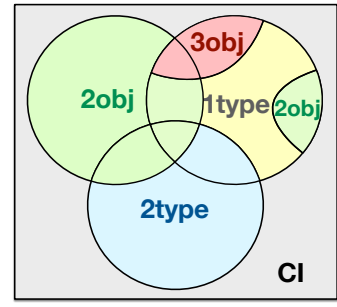

Unity

Fig. 2. An example for illustrating the idea of Unity. S1, S2 and S3 are three input selective context-sensitivity approaches. The four squares show the selected context-sensitivity information for the same program $P$ (i.e., which methods of $\mathrm{P}$ should be analyzed by what context-sensitivity variants) yielded by the three input selective approaches (S1, S2 and S3) and Unity respectively. For example, the square of S1 means: the S1 approach determines that the methods enclosed in the green circle will be analyzed by $20 b j$ while the remaining methods of $\mathrm{P}$ will be analyzed context-insensitively (i.e., $\mathrm{CI}$ ).

heavier context elements and longer context lengths, if possible. This counterintuitive proposal is based on the following insight.

Insight. Analyzing more methods context-sensitively may not incur an efficiency decline (sometimes it may even accelerate an analysis as more false data flows are pruned away), as long as the right methods are chosen. Avoiding scalability-threat methods is essential [Li et al. 2020; Smaragdakis et al. 2014], but the net number of methods analyzed context-sensitively is not.

As described in Section 2, each of the existing selective context-sensitivity approaches (henceforth, just "selective approaches") has its own insight (e.g., based on expert experience, parameterized heuristics, principled program patterns, etc.) to identify which methods are more likely useful for improving precision while not incurring much efficiency cost, when analyzed context-sensitively. Limited by the effectiveness of each insight, every approach may miss a collection of methods which are truly precision-useful and efficiency-friendly; however, this problem can be alleviated when combining more selective approaches to identify more target methods. As a result, we propose to simultaneously and context-sensitively analyze all the methods identified by a set of selective approaches, $S$. Although now possibly many more methods need to be analyzed context-sensitively, we still have a chance to obtain an analysis that is scalable even if we analyze them together, as each of these extra methods is determined as not a scalability threat by at least one approach in $S$. Hence, although it is hard to give one principle to accurately decide which methods, say M, are precision-useful but not scalability-threatening, our proposal naturally takes advantages of the insights of different selective approaches for identifying $M$ from different perspectives.

We illustrate the key idea of Unity in Figure 2. Assume we have three selective approaches S1, S2, and S3. Figure 2 depicts the selected context-sensitivity information determined by each approach for the same program P. For instance, after analyzing P, S1 reports that the set of methods in the green circle (in S1 of Figure 2) should be analyzed by 2obj and the remaining methods (the gray part in S1) need to be analyzed context-insensitively (CI). Similarly, we have the results for S2 and S3 in Figure 2, and in the case of S3, three context-sensitivity variants, 3obj, 2obj and 1 type are selected for different sets of methods.

What does Unity produce given the above three approaches and their selected context-sensitivity information? Briefly, Uni ty finds out the most precise configuration by reassigning context-sensitivity variants to the methods which are reported to be analyzed context-sensitively by at least two input approaches. For example, among the overlapped methods reported by S1 and S3, from the point 
of view of S1, some methods that should have been analyzed under 2obj, are now assigned to $3 \mathrm{obj}$ (color turns from green to red) as $30 \mathrm{bj}$ is more precise than $20 \mathrm{obj}$; for the rest of overlapped methods, they remain green (i.e., still be analyzed by $20 \mathrm{obj}$ ) as $2 \mathrm{obj}$ is more precise than 1 type (reported by S3). After applying similar treatment to other overlapped methods (with the disjoint ones unchanged), we get the output of Unity as in Figure 2. Note that the methods reported by all three input approaches (the middle part of the Unity figure) remain green as 2obj (reported by S1) is more precise than 2 type (by S2) and 1 type (by S3). Section 4 will detail the rules on how Unity deals with more input approaches with different context-sensitivity variants.

From the point of view of each selective approach, Unity modifies it by (1) adding many more methods (identified by other approaches) to be analyzed context-sensitively, and (2) upgrading the context-sensitivity variant to the most precise one for the methods which are also selected by other approaches. We have explained the insight for (1) at the beginning of Section 3.2, and the insight for (2) is similar: if a method $\mathrm{m}$ is reported to be analyzed under a certain context-sensitivity variant, cs, by an input approach $s$, this implies that according to the insight of $s, \mathrm{~m}$ is considered as not a scalability-threat, regardless of how costly cs is. Then, if the input approaches are reliable, we still have a chance to produce a scalable analysis, even if selecting the most precise cs. Later, experimental results further demonstrate the validity of the Unity insight: even if many more methods are analyzed context-sensitively and are under the most precise configuration (w.r.t. the input approaches), Unity is still able to scale for 12 out of 13 hard-to-analyze programs (with default settings).

However, there is no guarantee regarding the scalability of Unity. What can we do if Unity fails to scale? As a second try, a straightforward approach is to not make the analysis too costly. For example, if some overlapped methods $M$ are reported to be analyzed under 3obj and 1 type by different input approaches, what about considering the less precise (also less costly) one, i.e., 1 type? Nevertheless, this may introduce significant precision loss, and if $M$ is critical form improving precision, such treatment may make the analysis even less precise than the original one(s) guided by certain input approach(es) individually. To reap as much precision as possible while achieving good scalability, we employ the Relay technique.

The core insight of Relay is to divide the scalability burden of Unity into different passes (each pass is a run of selective context-sensitive pointer analysis) and the precision achieved by the former pass can be transmitted to and accumulated in the next pass. Based on this insight, we design two options of Relay, called Relay-01 and Relay-o2 that the former is more precise but less scalable than the latter. Figure 3 depicts the above idea with the same input selective approaches as in Figure 2. We introduce Relay-o1 first.

\subsection{Relay}

The first pass of Relay-01 in Figure 3 differs from Unity in that it only analyzes the methods (say M1) selected by S1 (enclosed in the circle with solid line) context-sensitively, with the remaining ones being analyzed under CI. Similarly, only the methods, say M2 (M3) selected by S2 (S3) are analyzed context-sensitively in pass 2 (pass 3). Thus, for each context-sensitive method of M1 (M2 or M3), as shown in the figure, Relay selects the same context-sensitivity variant for it as in Unity, namely, still the most precise configuration suggested by any of the combined approaches. For example, for M1 in pass 1, its context-sensitivity variants distribution is the same as the one of Unity in Figure 2 (i.e., the same subset of methods are assigned to $30 \mathrm{oj}$ while the remaining methods are assigned to $20 \mathrm{bj}$ ). For each pass of Relay, only a subset of methods of Unity are analyzed context-sensitively. Although this does not ensure better scalability than Unity, no more methods than the base approach are analyzed context-sensitively-only the same methods may be analyzed 


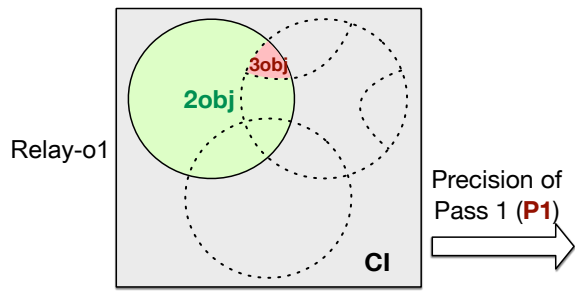

Pass 1

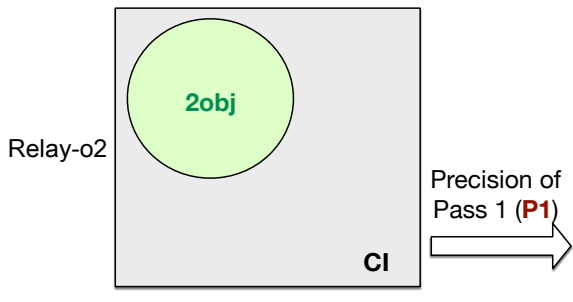

Pass 1

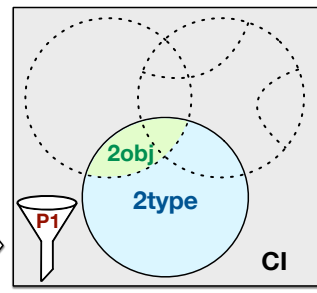

Pass 2

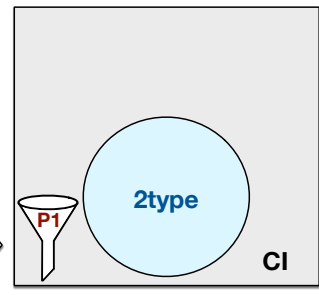

Pass 2

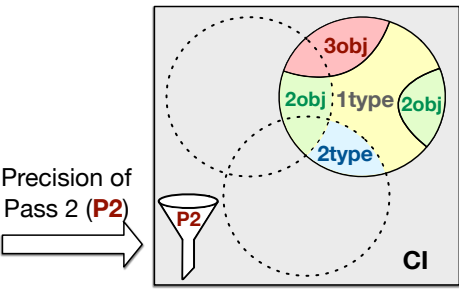

Pass 3

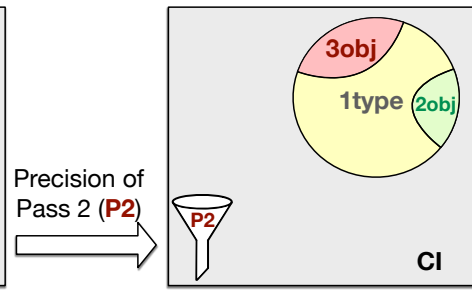

Pass 3

Fig. 3. Examples for illustrating the idea of Relay with the same input approaches setting as in Figure 2. Relay has two options, Relay-01 and Relay-02 and the former is more precise but less scalable than the latter. In each pass of both options, only the methods enclosed in the solid circles, say M, are analyzed context-sensitively (the dotted lines in Relay-o1 are to facilitate understanding). Both options adopt the same precision filtering mechanism, and their difference only lies in what context-sensitivity variants are assigned to $\mathrm{M}$.

with a more precise context. Therefore, the probability to select scalability-threat methods is low, making Relay more likely to scale compared to Unity.

After running the first pass, its precision, which is reflected in the points-to set for every variable, will be transmitted to the next pass (pass 2). Then, in pass 2, we can use the precision of pass 1 , i.e., the points-to information, to help soundly filtering the points-to sets for all variables when performing the analysis in pass 2 . For example, assume a variable $v$ points to three objects under $C I$ with its points-to set, say pt $(v)$, being $\{01,02,03\}$. After pass $1, \operatorname{pt}(v)$ becomes $\{01,03\}$ where 02 is identified as a spurious object by analyzing M1 context-sensitively. However, in pass 2, purely analyzing M2 under the contexts shown in Figure 3 cannot recognize that $v$ should not point to 02 . However, this imprecision does not matter, as the pt $(v)$ transmitted from pass 1 can be used to filter $\mathrm{pt}(\mathrm{v})$ during the analysis in pass 2, i.e., preventing 02 from being propagated to $\mathrm{pt}(\mathrm{v})$. Such filtering is safe as the pointer analysis in pass 1 is sound, so no true objects will be filtered away in Relay (this will be formally proven in Section 4).

Finally, although uncommon, what if Relay-01 is not scalable? After all, given a program, we hope to confidently rely on Unity-Relay for yielding precise pointer analysis results all the time. Under this context, we design Relay-02 as the last line of defense of Unity-Relay. Relay-o2 employs the same principle as Relay-01 with the exception that context-sensitivity variants (selected by input approach $S_{i}$ ) are not reassigned any more in each pass, say Pass ${ }_{i}$ (see Relay-o2 in Figure 3). In other words, Pass $i$ and the analysis guided by $S_{i}$, say $A_{i}$, adopt the same selective context sensitivity. The only difference between running the selective approaches independently is that precision accumulates, using the same filtering mechanism as in Relay-01. Thus, in Pass ${ }_{i}$, the points-to set for each variable is never larger than the one in $A_{i}$, resulting in less data propagation and faster analysis convergence. The only extra cost introduced in Relay-o2 is the process of filtering, which is small compared to the overhead of a context-sensitive pointer analysis, and easily offset by the aforementioned efficiency benefit. Thus, we can expect Relay-02 to scale as long 


\begin{tabular}{|c|c|c|c|c|c|c|c|}
\hline & & Variable & $\mathrm{CI}$ & S1 & S2 & S1\&S2 & Relay \\
\hline 1 & $x \cdot f=a$; & $a$ & 01 & 01 & 01 & 01 & 01 \\
\hline 2 & $b=y \cdot f ;$ & $x$ & 02 & 02 & 02 & 02 & 02 \\
\hline 3 & $\ldots$ & $\mathrm{y}$ & 02,03 & 03 & 02,03 & 03 & 03 \\
\hline 4 & $p \cdot f=a$; & $\mathrm{p}$ & 04 & 04 & 04 & 04 & 04 \\
\hline & $b=q . f ;$ & $q$ & 04,05 & 04,05 & 05 & 05 & 05 \\
\hline & & b & 01 & 01 & 01 & 01 & \\
\hline
\end{tabular}

Fig. 4. Example for illustrating precision intervention in Relay. Specifically, $b$ is supposed to be a null pointer at run time, and only Relay can analyze it precisely in this case.

as $\mathrm{A}_{i}$ scales. We will further discuss the scalability of Relay-o2 in Section 4. Note that Relay-o2 is fundamentally different from merely intersecting the points-to results of all pointer analyses guided by the input approaches. In Relay-02, there is early precision intervention of each analysis pass to the next: the pointer analysis in each pass is filtered by the points-to results produced by the previous passes on the fly, likely preventing the computation of more spurious value flows during the analysis. As a result, Relay-02 is more precise than simply intersecting the final points-to sets after individually running each guided pointer analysis without such precision intervention.

We next use an example, in Figure 4, to illustrate the benefit of precision intervention. The left side of Figure 4 is an example code snippet, where the value of $a$ is stored into $x . f$ and $p . f$ (through lines 1 and 4), and b loads values from y.f and q.f (through lines 2 and 5). The table on the right side contains the points-to sets of the variables in the code snippet under different pointer analyses. Column CI gives the points-to sets produced by a context-insensitive pointer analysis, and we consider two selective context-sensitive pointer analyses, S1 and S2, which identify o2 and o4 as spurious objects for variables y and q, respectively. If we simply intersect the points-to results of S1 and S2, then we can eliminate spurious objects for $y$ and $q$, as shown in column S1\&S2. However, b still points to the spurious object 01 under S1\&S2, as 01 can flow to $b$ through either line 2 or line 5: both S1 and S2 consider 01 to be valid for $b$, for different reasons each. Among the analyses in the table, only Relay can block the two flows (through lines 2 and 5) simultaneously. In Relay, with $\mathrm{S} 1$ as pass 1 and S2 as pass 2, S2 identifies o4 as spurious for $\mathrm{q}$ so that the flow through line 5 can be blocked (as $p$ and $q$ are not aliased). Further benefiting from the precision intervention, the flow through line 2 is also blocked as pass 2 also incorporates the result from pass 1, i.e., 02 is identified as spurious for $y$ by $\mathrm{S} 1$ (so that $\mathrm{x}$ and $\mathrm{y}$ are not aliased). As a result, $\mathrm{o} 1$ is identified as spurious for $b$ in pass 2. (The points-to set of $b$ is empty, based on the fragment and example values shown, but other program statements could give it values, orthogonally to the example-e.g., by setting y.f or q.f.)

In the overall Relay mechanism, Relay-01 and Relay-02 apply independently to analysis passes and are mixed as necessary for scalability. The two Relay variants can be seen as the best and worst precision options, and there is design space between them for making precision and efficiency trade-offs. In our design, precision is the first priority, so for every Pass ${ }_{i}$, we first attempt Relay-o1. If Relay-01 is not scalable, we select Relay-02 (which scales if the base approach does), and repeat the same scheme (i.e., first trying Relay-01, then Relay-02 if it fails) to Pass $s_{i+1}$, etc. until all $n$ passes complete (assuming there are $n$ input selective approaches).

The running order for different passes of Relay does not affect the soundness and precision guarantees of Relay (including both Relay-01 and Relay-02) and also the scalability potential of Relay-02 (as proved and discussed in Section 4.4). In other words, in practice, regardless of the pass ordering, users can rely on Relay to produce an analysis that is more precise than the one (say 
A) guided by any input selective approach, and can expect Relay-02 to scale as long as A scales. However, the pass ordering may affect the final precision of Relay (although experimental results show that such effect is very minor). For example, assume two variables $v 1$ and $v 2$ point to $\{01,02\}$ and $\{03,04\}$ respectively under CI. Pass 1 identifies 02 as a spurious object for $v 1$, and pass 2 can identify 04 as a false object if and only if it is aware that $v 1$ does not point to 02 with the help of the result from pass 1 . But when we swap the order of pass 1 and pass 2 , v2 may still point to 04 (actually a spurious object) at the conclusion of all passes, resulting in different precision. We will discuss the pass ordering issue using the experimental results in Section 6.2.

\section{FORMALISM AND PROPERTIES}

We formalize the Unity-Relay framework, prove its key properties, and discuss its important features. Specifically, we detail how to unify various context-sensitivity approaches with different context elements and lengths, and how to guide selective context-sensitive pointer analysis in each pass to filter spurious objects using the results from the previous pass, while achieving soundness and precision guarantees.

\subsection{Domain and Notations}

Our notation is shown in Figure 5. For formalization purposes, two domains of context elements, $\mathbb{K}$ and $\mathbb{E}$ are introduced. The former denotes the kinds of context elements, i.e., obj for object sensitivity, type for type-sensitivity and call for call-site sensitivity (identified by their locations in the program) while the latter denotes the concrete context elements forming each context $c \in \mathbb{C}$. For instance, in object sensitivity, $c$ consists of a list of heap objects (allocation sites) $o_{i} \in \mathbb{E}$. Each context-sensitivity variant $c s \in \mathbb{C}$ can be expressed as a pair of context length $l$ and a kind of context element $k$, denoted by $l k$, e.g., 2obj or 1 type, hence we have $\mathbb{C}=\mathbb{N} \times \mathbb{K}$, where $\mathbb{N}$ denotes the natural numbers.

$p t$ and $f p t$ denote the analysis results. $p t(c, x)$ represents the points-to set of variable $x$ under context $c$. $f p t\left(c, o_{i}, f\right)$ represents the points-to set of instance field $f$ of an abstract object $o_{i}$ under context $c$. The objects in the points-to sets of $p t$ and $f p t$ are also qualified by contexts, i.e., heap contexts.

gen $_{c s}$ denotes the function that generates contexts according to the length and kind of context elements of $c s$, which will be further explained in Section 4.2. contextsOf maps a method to a set of contexts under which the method is analyzed. selectCS is the core of selective context sensitivity, which selects a proper context-sensitivity variant $c s$ for each method. We use $S$ to denote a set of selective approaches, indexed by subscript: $S_{i}$. If a pointer analysis PA is guided by a selective approach $S_{i}$, we refer to it as PA-S $S_{i}$

\subsection{Selective Context-Sensitive Pointer Analysis}

We present a formulation of general selective context-sensitive pointer analysis in Figure 6, which covers five basic statements: object allocation ([NEw]), local assignment ([Assign]), field load ([LoAD]) and store ([Store]), and method call ([CALL]). Similar to [Sridharan et al. 2013; Tan et al. 2016], we elide the rules for static members and arrays, as the former is straightforward and the latter can be modeled as load and store to an artificial field of each array. In Figure 6, the premises within boxes are only related to Unity-Relay, and thus can be ignored for now.

For each rule in Figure 6, $m$ denotes the method containing the corresponding statement (in blue color). Here we only explain $[\mathrm{NEw}]$ and $[\mathrm{CALL}]$ as the other three rules are standard.

In context-sensitive pointer analysis, an abstract object is typically represented by a heap context and the label of the allocation site, as in our [NEw] rule. For simplicity, we directly use the context of the method containing the allocation site (i.e., $c$ ) as the heap context for the created abstract 


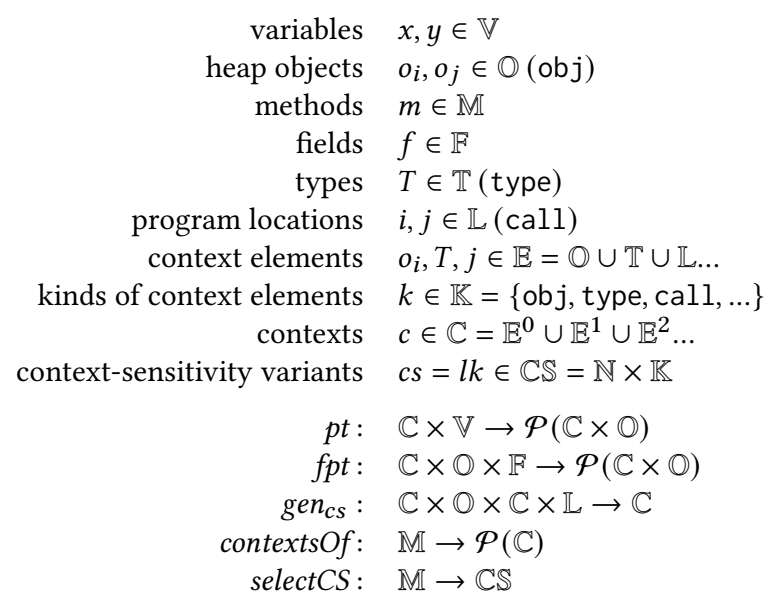

Fig. 5. Domain and notations.

object. This is a bit different from some existing literature [Jeon et al. 2018; Milanova et al. 2005; Smaragdakis and Balatsouras 2015; Sridharan et al. 2013], which derives the heap context from the method context-e.g., allowing to cut out part of the method context, as the heap context may be shorter. We omit this general treatment in the formal model, for simplicity of illustration purposes. Our implementation supports the more general, full choice in selecting heap contexts.

In [CALL], function dispatch $\left(o_{i}, g\right)$ is applied to resolve the virtual dispatch of $g$ on receiver object $o_{i}$ to the callee $m^{\prime}$.

Without loss of generality, we model the results of each selective context-sensitivity approach as a function selectCS, which is invoked to select a proper context-sensitivity variant $c s$ for $m^{\prime}$. Actually, traditional context sensitivity can be seen as a special case of selectCS, which returns the same $c s$ for all methods.

Each context-sensitivity variant $c s$ corresponds to a function gen ${ }_{c s}$ (see Figure 5), which generates contexts for target methods based on the information available at the call site. For generality, as

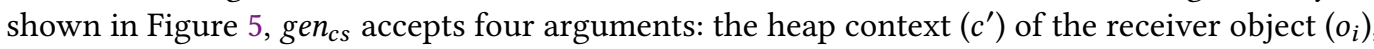
the context of caller $m^{\prime}(c)$, and the call site $(j)$, so that it can generate contexts for different contextsensitivity variants, including object, type, and call-site sensitivity. The generated context for the target method is denoted $c^{t}$.

We use $m_{\text {this }}^{\prime}$ to represent the this pseudo-variable of method $m^{\prime}, m_{p k}^{\prime}$ to represent the $k$-th parameter of $m^{\prime}$, and define for every $m^{\prime}$ a variable $m_{r e t}^{\prime}$ holding all its return values. The rest of [CALL] propagates arguments and return values between caller in context $c$ and callee in context $c^{t}$.

\subsection{Unity}

We formalize Uni ty by defining its C.S. selector (Figure 1), i.e., selectCS in Figure 6. Based on a set of input selective approaches $S$, Unity selects the most precise context-sensitivity variant for each method. We define $\leq$, a binary relation of precision between two context-sensitivity variants. If $c s$ is less precise than (or as precise as) $c s^{\prime}$, we write $c s \leq c s^{\prime}$.

The precision of different context-sensitivity variants can be compared based on their lengths and kinds of context elements. The impact of length on precision is well understood, i.e., longer length has better precision (e.g., 2obj $\leq 30 b j$ ).

Are context-sensitivity variants with different kinds of elements comparable in terms of precision? The answer is yes, but not always. Here, we say two variants are comparable means that one of them 


$$
\begin{aligned}
& \frac{i: x=\text { new } T() \quad c \in \text { contexts } O f(m)}{\left\langle c, o_{i}\right\rangle \in p t(c, x)}[\mathrm{New}] \\
& x=y \quad c \in \text { contexts } O f(m) \\
& \frac{\left\langle c, o_{i}\right\rangle \in p t(c, y) \quad o_{i} \in \operatorname{ppt}(x)}{\left\langle c, o_{i}\right\rangle \in p t(c, x)} \text { [Assign] } \\
& x=y \cdot f \quad c \in \text { contexts } O f(m) \quad\left\langle c^{\prime}, o_{i}\right\rangle \in p t(c, y) \\
& \left\langle c^{\prime \prime}, o_{j}\right\rangle \in \operatorname{fpt}\left(c^{\prime}, o_{i}, f\right) \quad o_{j} \in \operatorname{ppt}(x) \\
& \left\langle c^{\prime \prime}, o_{j}\right\rangle \in p t(c, x) \\
& x . f=y \quad c \in \text { contexts } O f(m) \\
& \frac{\left\langle c^{\prime}, o_{i}\right\rangle \in p t(c, x) \quad\left\langle c^{\prime \prime}, o_{j}\right\rangle \in p t(c, y)}{\left\langle c^{\prime \prime}, o_{j}\right\rangle \in f p t\left(c^{\prime}, o_{i}, f\right)} \text { [Store] } \\
& j: x=y \cdot g\left(a_{1}, \ldots, a_{n}\right) \quad c \in \operatorname{contextsOf}(m) \\
& \left\langle c^{\prime}, o_{i}\right\rangle \in p t(c, y) \quad o_{i} \in \operatorname{ppt}\left(m_{\text {this }}^{\prime}\right) \\
& m^{\prime}=\operatorname{dispatch}\left(o_{i}, g\right) \\
& c s=\operatorname{selectCS}\left(m^{\prime}\right) \quad c^{t}=\operatorname{gen}_{c s}\left(c^{\prime}, o_{i}, c, j\right) \\
& \left\langle c^{a}, o_{a}\right\rangle \in p t\left(c, a_{k}\right), 1 \leq k \leq n \quad o_{a} \in \operatorname{ppt}\left(m_{p k}^{\prime}\right) \\
& \frac{\left\langle c^{r}, o_{r}\right\rangle \in p t\left(c^{t}, m_{r e t}^{\prime}\right) \quad o_{r} \in \operatorname{ppt}(x)}{c^{t} \in \text { contextsOf }\left(m^{\prime}\right) \quad\left\langle c^{\prime}, o_{i}\right\rangle \in p t\left(c^{t}, m_{t h i s}^{\prime}\right)} \text { [CALL] } \\
& \left\langle c^{a}, o_{a}\right\rangle \in p t\left(c^{t}, m_{p k}^{\prime}\right) \quad\left\langle c^{r}, o_{r}\right\rangle \in p t(c, x)
\end{aligned}
$$

Fig. 6. Rules for selective context-sensitive pointer analysis. The premises enclosed in boxes are specific to Unity-Relay-based pointer analysis.

is always more precise than (or as precise as) the other one. For such comparison, we introduce notation $k \sqsubseteq k^{\prime}$ to represent that using context element $k$ is less precise than (or as precise as) $k^{\prime}$.

The variants of some kinds of context elements are comparable, e.g., obj and type. As type is a coarser context abstraction over (and always less precise than) object [Smaragdakis et al. 2011], we have type $\sqsubseteq \mathrm{obj}$, and accordingly, $l$ type $\leq l o b j$. In other cases, such as $l o b j$ and $l$ call, their precision are theoretically incomparable. We will discuss how to handle such cases at the end of this section. For now, we assume that all concerned context-sensitivity variants are comparable.

Now we define $c s \leq c s^{\prime}$ :

$$
\begin{array}{r}
c s \leq c s^{\prime} \text { iff } c s=\mathrm{CI} \vee\left(l \leqslant l^{\prime} \wedge k \sqsubseteq k^{\prime}\right) \\
\text { where } c s=l k \text { and } c s^{\prime}=l^{\prime} k^{\prime}
\end{array}
$$

Formula (1) states that any context sensitivity variant is not less precise than CI, and $c s \leq c s^{\prime}$ only when both length and kind of context elements of $c s$ are at most as precise as those of $c s^{\prime}$.

Next we can formalize the C.S. selector of Unity as a function selectCS-unity $S^{S}(m)$ :

$$
\text { selectCS-unity } S^{S}(m)=\max \leq_{S_{i} \in S} \text { selectCS }(m)
$$

Based on $\leq$, $\max \leq_{S_{i} \in S}$ chooses the most precise $c s$ from a set of selective approaches $S$ for method $m$. selectCS $S_{i}$ corresponds to the selector function for $S_{i}$. By specifying selectCS in [CALL] to selectCS-unity ${ }^{S}$, we get a pointer analysis guided by Unity, i.e., PA-Unity ${ }^{\text {. }}$. 


\section{Theorem 4.1 (Soundness of Unity). PA-Unity ${ }^{S}$ is sound.}

Proof. By rules in Figure 6, PA-Unity ${ }^{S}$ is a standard Andersen-style pointer analysis, and the only difference from other selective context-sensitive pointer analyses is selectCS. Selecting different contexts for methods affects precision but not soundness [Jeon et al. 2018; Jeong et al. 2017; Li et al. 2018b, 2020; Smaragdakis et al. 2014]. Hence PA-Unity ${ }^{S}$ is sound.

Following existing literature [Kastrinis and Smaragdakis 2013; Li et al. 2018b; Lu and Xue 2019; Minseok Jeon and Oh 2020], contexts in points-to results are ignored when measuring the precision of context-sensitive pointer analyses. To this end, we use $\overline{p t}_{\mathrm{PA}}(x)$ to represent the points-to set of variable $x$ in pointer analysis $\mathrm{PA}$, without contexts, e.g., if $p t(c, x)=\left\{\left\langle c^{i}, o_{i}\right\rangle\right\}$ and $p t\left(c^{\prime}, x\right)=\left\{\left\langle c^{j}, o_{j}\right\rangle\right\}$ in PA, then $\overline{p t}_{\mathrm{PA}}(x)=\left\{o_{i}, o_{j}\right\}$.

Definition 4.2 (Precision of Pointer Analysis). Given two sound pointer analyses PA and $\mathrm{PA}^{\prime}, \mathrm{PA}$ is at most as precise than $\mathrm{PA}^{\prime}$, denoted $\mathrm{PA} \leq \mathrm{PA}^{\prime}$, if for each variable $x: \overline{p t}_{\mathrm{PA}}(x) \supseteq \overline{p t}_{\mathrm{PA}^{\prime}}(x)$.

(We informally also use the convenient forms "less precise", as a synonym of "at most as precise", and "more precise" as a synonym of the converse. "Less than or equally" and "more than or equally" would have been more accurate, but inconvenient.)

We define the precision of a pointer analysis based on the points-to sets of each variable, as they are the most important results of pointer analysis and used by virtually all its clients.

Theorem 4.3 (Precision of Unity). Given a set of selective approaches $S, \forall S_{i} \in S: \mathrm{PA}-S_{i} \leq$ PA-Unity ${ }^{S}$.

Proof. By Equation (2), the cs selected by selectCS-unity $S^{S}$ for each method is always more precise than the one selected by any $S_{i} \in S$. This ensures that for each variable $x$ in any PA- $S_{i}$, $\overline{p t}_{\mathrm{PA}-S_{i}}(x) \supseteq \overline{p t}_{\mathrm{PA}-U n i t y}(x)$. Hence, $\forall S_{i} \in S:$ PA-S $S_{i} \leq$ PA-Unity ${ }^{S}$.

\subsection{Relay}

Given a set of selective approaches $S$, in Relay, we run $|S|$ pointer analysis passes to divide the scalability burden into different passes (Section 3.3). Now we formalize the C.S. selector (Figure 1) of the two options of Relay (Relay-01 and Relay-02) for any pass . $_{i}$

We define the C.S. selector of Relay-o1 for pass $_{i}$ (denoted by selectCS-relay $y_{i}^{S}$ ), as follows:

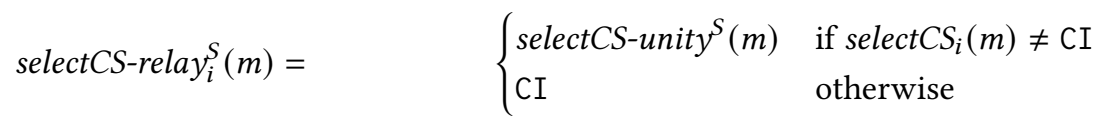

For a method $m$, if selectCS $S_{i}$ (result of the $i$-th selective approach) selects a non-CI variant for $m$, then selectCS-relay $y_{i}^{S}$ leverages selectCS-unity $S^{S}$ to select the most precise $c s$, otherwise, CI is selected, as illustrated in Figure 3.

In Relay-o2, to ensure scalability in pass $_{i}$, as explained in Section 3, we use the same context selector function as $S_{i}$ :

$$
\text { selectCS-relay } S_{i}(m)={\operatorname{select} C S_{i}}_{(}(m)
$$

In each pass of Relay (except pass ${ }_{1}$ ), we apply filtering on the points-to sets of variables based on the points-to results from the previous pass, to improve precision by reducing spurious data flows. Such filtering is formalized as the premises in boxes of [Assign], [LoAD] and [CALL] (Figure 6), where $\operatorname{ppt}(x)$ denotes the points-to set of variable $x$ (without contexts) in the previous analysis pass ( $p p t$ reads as "previous points-to set"). We ignore contexts in $p p t$ as the contexts of different passes may not be possible to match. These boxed premises ensure that if the previous analysis concludes that variable $x$ does not point to object $o_{i}$, then all $\left\langle_{-}, o_{i}\right\rangle$ will be filtered out from $p t\left(_{-}, x\right)$ in the 
current (and future) passes, as they are identified as spurious points-to information by previous analyses.

Filtering prevents spurious objects from being propagated to variables. [NEw] does not propagate spurious objects (for statement $i: x=$ new $T\left(\right.$ ), $x$ must point to $o_{i}$ ). [STORE] propagates objects to instance fields (not variables), and the stored objects can only be accessed by a load operation. Then with the filtering by ppt in [LOAD], spurious objects from instance fields will not be propagated to other variables. Thus we do not need to apply filtering for [New] and [STORE].

With filtering, the precision achieved in each pass can be transmitted to and accumulated in later passes.

Lemma 4.4 (SOUndness of Filtering). In Figure 6, if ppt(_) are the results of a sound pointer analysis $\mathrm{PA}$, then the resulting filtered pointer analysis $\mathrm{PA}^{\prime}$ is also sound.

Proof. By [Assign], [LOAD], and [CALL], the filtering only removes the points-to results which are absent in PA. Since PA is sound, it will not filter out true points-to results. Hence the filtering based on PA does not affect soundness in $\mathrm{PA}^{\prime}$.

Theorem 4.5 (Soundness of Relay). For any pass $s_{i}$ in Relay, PA-Relay ${ }_{i}^{S}$ is sound.

Proof. For any pass ${ }_{i}$ in Relay, we assume that PA-Relay ${ }_{i}^{S}$ may use any approach in $S$, i.e., the approaches in $S$ can be arranged in any order. By the rules in Figure 6, PA-Relay ${ }_{i}^{S}$ without pointer analysis filtering is sound (refer to the proof of Theorem 4.1). As pass p $_{1}$ of Relay does not have filtering, PA-Relay ${ }_{1}^{S}$ is sound (regardless of the approach it uses). The filtering of PA-Relay ${ }_{i}^{S}$ is based on the results of PA-Relay ${ }_{i-1}^{S}$. Thus, by Lemma 4.4, no matter what approaches PA-Relay ${ }_{i-1}^{S}$ and PA-Relay ${ }_{i}^{S}$ use (i.e., the order of approaches is irrelevant), if PA-Relay ${ }_{i-1}^{S}$ is sound, PA-Relay ${ }_{i}^{S}$ is sound. By induction, for any $\operatorname{pass}_{i}, \mathrm{PA}-\mathrm{Relay}_{i}^{S}$ is sound.

Theorem 4.6 (Precision of Relay). Given a set of selective approaches $S, \forall S_{i} \in S:$ PA- $S_{i} \leq$ PA-Relay ${ }_{i}^{S} \leq$ PA-Relay ${ }_{|S|}^{S}$ (the last pass of Relay) for both Relay-01 and Relay-02.

Proof. Similarly to the proof of Theorem 4.5, we assume that PA-Relay ${ }_{i}^{S}$ may use any approach in $S$. By Equations (3) and (4), for each method, the cs selected by selectCS-relay $y_{i}^{S}$ (of both Relay-o1 and Relay-02) is more precise than that selected by selectCS $S_{i}$, thus, $\forall S_{i} \in S: \mathrm{PA}-S_{i} \leq \mathrm{PA}-\mathrm{Relay}_{i}^{S}$ regardless of what approach $S_{i}$ is. By pointer analysis filtering, each variable $x$ in pass ${ }_{i}$ must point to fewer (or the same) objects than $x$ in pass $_{i-1}$, i.e., PA-Relay ${ }_{i-1}^{S} \leq \mathrm{PA}-R e l a y_{i}^{S}$; in other words, each pass is more precise than all its previous passes, no matter what approaches these passes use (i.e., the order of approaches is irrelevant). As PA-Relay ${ }_{|S|}^{S}$ is the last pass of Relay, we have

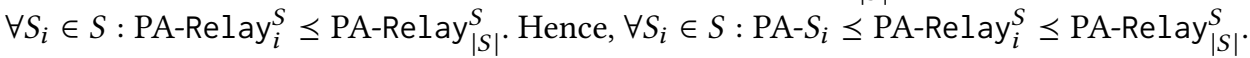

Interestingly, Theorem 4.6 holds for Relay-02 regardless of whether the context-sensitivity variants selected by selectCS-relay $y_{i}^{S}$ are comparable or not. Since by Equation (4), Relay-o2 selects the same context-sensitivity variants as the selective approach $S_{i}$ in pass ${ }_{i}$, and further by the precision accumulation (via filtering), PA-Relay ${ }_{i}^{S}$ is at least as precise as (practically more precise than) PA- $S_{i}$. Accordingly, the last pass of Relay-o2 is at least as precise as any PA- $S_{i}$.

As for Unity and Relay-01, in the case that some selected variants are theoretically incomparable, e.g., 2obj and 2call, their precision guarantee does not hold in theory. But in practice, users can choose an appropriate one empirically. E.g., 2obj is typically much more precise than 2call [Kastrinis and Smaragdakis 2013; Tan et al. 2016]. In this way, we can still obtain from Unity-Relay a pointer analysis that is likely more precise than any $S_{i}$ in practice. 
Scalability. Relay-02 is the last line of defense of Unity-Relay: given a set of selective approaches $S$, we can expect Relay-o2 to scale as long as $\forall S_{i} \in S:$ PA- $S_{i}$ scales. With conventional approaches, when context-sensitively analyzing a set of scalability-threat methods, (1) the size of context-sensitive points-to relations may become large (thus needing much more time to handle in every analysis iteration), and (2) the analysis may need many more iterations to reach a fixed point. The simultaneous effects of (1) and (2) make the analysis complexity blow up, becoming unscalable. However, for Relay-02, in each pass the context-sensitivity variant selected is the same as that of PA- $S_{i}$ for every method, and due to the filtering mechanism, the points-to set for every variable is never larger than the one in $\mathrm{PA}-S_{i}$ (note that this holds regardless of the pass ordering in Relay-02); therefore, in Relay-02, there will be no more data propagated in each iteration and no more iterations until convergence. Thus Relay-02 scalability is ensured, as long as each PA- $S_{i}$ scales. The only extra cost introduced in Relay-02 is its filtering process and its essence is querying whether an element (object) belongs to a set (points-to set), which can be implemented efficiently, and unlike (1) and (2) described above, it is not a key factor in making a pointer analysis unscalable. The experimental results in Section 6.2 further validate the scalability of Relay.

Other Context Abstractions for Selective Context Sensitivity. Our current formalism only focuses on the widely-used method-level selective approaches, i.e., it assumes that the input selective approaches choose context based on the target method $(m$ in selectCS $(m)$ ). Still, the idea of Uni ty-Relay also applies to other context abstractions for selective context sensitivity. For example, introspective analysis [Smaragdakis et al. 2014] selects context based on call sites (i.e., different context-sensitivity variants for different call sites). To support such a selective approach, we only need to change the input domain of select $C S$ to further include call sites, and the context selection policy of Unity-Relay remains the same. As another alternative, Lu and Xue [2019] select context on a per-variable basis. The Uni ty-Relay idea applies seamlessly, but at the granularity of variables, not methods, i.e., one should see the circles in Figures 2 and 3 as selected variables.

\section{BATON}

As a proof-of-concept, we introduce BATON, an instantiation of the Unity-Relay framework. Given plenty of existing selective context sensitivity approaches [Hassanshahi et al. 2017; Jeon et al. 2019; Jeong et al. 2017; Li et al. 2018a,b, 2020; Lu and Xue 2019; Minseok Jeon and Oh 2020; Oh et al. 2014, 2015; Smaragdakis et al. 2014; Wei and Ryder 2015], the design space of Uni ty-Relay is large. For more precision improvement, the chosen approaches should be diverse so that they could cover precision-useful methods from different perspectives; and they should exhibit good scalability, as explained in Section 3.3. Accordingly, BATON considers three such approaches as inputs: one ad-hoc approach based on expert experience, called COLLECTION [WALA 2018], and two state-of-the-art approaches, called ZIPPER $^{e}$ [Li et al. 2020] and SCALER [Li et al. 2018b].

Collection (or container) methods are important to pointer analysis, as a huge amount of objects may flow to and are merged in them, if the collection is not being analyzed context-sensitively. Thus, in pointer analysis frameworks such as WALA [WALA 2018], the experts provide an option to analyze only collection methods (in JDK) context-sensitively for good precision (with also good scalability). Inspired by [WALA 2018], in CoLLEction, we apply 3obj to the collection methods whose declaring classes implement interfaces java.util. Collection and java.util. Map in both application and library code.

ZIPPER $^{e}$ (short for ZIPPER express) [Li et al. 2020] is a variant of a selective approach called ZIPPER [Li et al. 2018a] which identifies precision-useful methods based on principled precision-loss patterns [Li et al. 2020]. By exploiting the patterns, ZIPPER ${ }^{e}$ adopts simple but effective heuristics to further exclude the scalability-threat methods. As a result, by applying $20 \mathrm{obj}$ only to the finally 
selected methods (with the others being analyzed context-insensitively), compared to ZIPPER, ZIPPER $^{e}$ substantially improves efficiency with similar precision.

SCALER [Li et al. 2018b] leverages the object allocation graph in [Tan et al. 2016] to estimate the context-sensitive points-to sizes that would be needed for each method. Then it selects an appropriate context-sensitivity variant (2obj, 2type, 1 type or CI) for each method, while keeping the overall points-to size bounded (to a quantity that represents the memory capacity available for running the analysis), resulting in good scalability.

For a given program $\mathrm{P}$, BATON applies the above three approaches to analyze $\mathrm{P}$ to obtain their context-sensitivity variants selected for each method of $P$. Based on these results, BAToN produces new context-sensitivity configurations according to the Unity and Relay principles. In particular, in Relay, we run COLLection, ZIPPER ${ }^{e}$ and SCALER in this order. We switch between Relay-o1 and Relay-02, choosing the more precise option (Relay-01) if it scales, per the discussion of Section 3.

As all context-sensitivity variants selected by COLLECTION, ZIPPER ${ }^{e}$ and SCALER are comparable in precision, by Theorems 4.3 and 4.6, we can expect BATON to yield more precise results than all of them in practice. This will be further validated in Section 6.

\section{EVALUATION}

This section examines how BATON performs when addressing the challenging research problem raised in Section 1: "Given reasonably long time, can we achieve precise pointer analysis results for hard-to-analyze programs, for which traditional context-sensitive analyses fail to scale, and selective context-sensitive approaches scale but with limited precision?". We investigate the following research questions:

RQ1. Given that BATON (Unity) picks the "most precise configuration" based on the input selective approaches, how does this design scale for hard-to-analyze programs in practice? How does it fare against state-of-the-art analyses in terms of the precision gain that we aim for?

RQ2. In the cases for which BATON (Unity) fails to scale, how does BATON (Relay), as the second punch of Unity-Relay, perform in terms of scalability and precision?

Experimental Settings. We conduct all experiments on a machine with an Intel Xeon $2.2 \mathrm{GHz} \mathrm{CPU}$ and 128GB of memory. All pointer analyses are performed on Doop [Bravenboer and Smaragdakis 2009], the state-of-the-art pointer analysis framework for Java (with the version published as the artifact of [Smaragdakis et al. 2014]). All pointer analyses adopt the same reflection handling configuration for the same program. Specifically, for each program, we first run the dynamic reflection analysis tool TAMIFLEX [Bodden et al. 2011] and its results are used if TAMIFLEX analyzes the program successfully; otherwise (if TAMIFlex throws exceptions), we use Doop's default reflection analysis setting. Time budget is set to 2 hours for each analysis. In evaluation, all benchmarks are analyzed with a large Java library: OpenJDK 1.6.0_24, which is widely used in recent work [Jeon et al. 2019, 2018; Li et al. 2018a, 2020; Minseok Jeon and Oh 2020].

Hard-to-analyze Programs. We consider 13 large and complex Java programs as our benchmarks, including all the hard-to-analyze programs in the standard DaCapo benchmarks [Blackburn et al. 2006] and recent literature for Java pointer analysis [Jeon et al. 2019, 2018; Jeong et al. 2017; Kastrinis and Smaragdakis 2013; Li et al. 2018b, 2020; Minseok Jeon and Oh 2020; Smaragdakis et al. 2014], for which traditional 2obj fails to scale within time budget ( 2 hours). To our knowledge, this is the largest set of hard-to-analyze programs evaluated in related literature.

Precision Metrics. To thoroughly measure precision, we consider the most complete set of precision metrics that were used in recent literature [Jeon et al. 2019, 2018; Jeong et al. 2017; Kastrinis and 
Smaragdakis 2013; Li et al. 2018a,b, 2020; Lu and Xue 2019; Minseok Jeon and Oh 2020; Smaragdakis et al. 2014]. It consists of three general precision metrics, i.e., the total size of points-to sets for all variables (VarPts), the average size of points-to set per variable (AvgPts), and the number of all may-alias variable pairs (Aliases); and four independently useful client analyses that are often adopted in the literature to measure precision. The clients include a cast-resolution analysis (with the metric being the number of cast operations that may fail, denoted FCasts), a devirtualization analysis (with the metric being the number of virtual call sites that may have multiple call targets, denoted PCalls), a method reachability analysis (with the metric being the number of reachable methods, denoted RMtds), and a call-graph construction analysis (with the metric being the number of call graph edges, denoted CEdges).

State of the art. The nature of Unity-Relay enables BATON to take advantage of the precision achieved by any selective approach (as input), thereby whatever the state-of-the-art is, BATON can achieve practically better precision (Theorems 4.3 and 4.6). In this context, we compare against two state-of-the-art selective approaches, ZIPPER ${ }^{e}$ [Li et al. 2020] and SCALER [Li et al. 2018b] (also adopted by BATON as inputs in Section 5), to evaluate how much more precision BATON can achieve. ZIPPER ${ }^{e}$ and SCALER are open-source tools that try to maximize the precision while not threatening scalability, and have been demonstrated to be significantly more precise than other selective approaches [Smaragdakis et al. 2014]. We do not consider ZIPPER [Li et al. 2018a] as it fails to scale for all our benchmarks.

\subsection{RQ1. Precision and Scalability of BATON (Unity).}

For ZIPPER ${ }^{e}$ and SCALER, we use their default configurations as in Li et al. [2018b, 2020], which are well-tuned and achieve good precision and efficiency trade-offs. As part of BATON, we also include COLLECTION for comparison. To provide a sense of how precise the pointer analyses (guided by these selective approaches) are, we consider CI as a baseline.

Figure 7 shows graphically the precision improvement for a single metric: Aliases (i.e., may-alias variable pairs). Table 1 presents the results for all analyses, in full detail. For each program, there are five rows of data, corresponding to the five analyses evaluated. Columns 3-9 list the seven precision metrics explained above, and the rightmost column shows the analysis time in seconds. Note that ZIPPER ${ }^{e}$, SCALER and BATON (Uni ty) require a pre-analysis to select contexts, however, these computations are very fast (from seconds to minutes) and are negligible compared to pointer analysis time, thus the pre-analysis time is elided in our evaluation. Next, we examine the detailed results in Table 1. For all numbers in the table, lower is better.

Precision. From Table 1, we can see that ZIPPER ${ }^{e}$, SCAlER and Collection are much more precise than CI. For BATON (Uni ty), as expected, it achieves better precision than all these three analyses for all precision metrics for all programs in Table 1. This result also validates Theorem 4.3. The precision improvements are significant in general, and are striking for some programs such as hsqldb and heritrix. Considering that the compared analyses are already highly precise, such precision improvements made by BATON (Unity) are impressive. BATON (Unity) performs well especially for VarPts, AvgPts and Aliases, which are the most important outputs of pointer analysis, necessary for numerous clients. The precision improvements for PCalls and RMtds are not as remarkable as other metrics. Actually, existing work has shown that the precision improvement of a pointer analysis has less impact on these two clients compared to others [Kastrinis and Smaragdakis 2013]. This can also be observed in the experimental results from recent selective context-sensitive pointer analysis work [Jeon et al. 2019; Jeong et al. 2017; Li et al. 2020]. Finally, due to high precision, BATON (Unity) can avoid spending time on propagating many spurious data flows. Thus, sometimes, it can run even faster than individual analyses (COLLECTION, ZIPPER ${ }^{e}$ or SCALER) as discussed below. 
Table 1. Precision and performance metrics for context-insensitive (CI) and selective context-sensitive pointer analyses guided by Collection, ZIPPER ${ }^{e}$, SCALER, and BATON (Unity) respectively. For all numbers, lower is better.

\begin{tabular}{|c|c|c|c|c|c|c|c|c|c|}
\hline Program & Analysis & VarPts & AvgPts & Aliases & FCasts & PCalls & RMtds & CEdges & Time (s) \\
\hline \multirow{5}{*}{ hsqldb } & CI & $5,120,447$ & 53.4 & $54,502,418$ & 1,662 & 1,592 & 11,486 & 63,790 & 59 \\
\hline & COLLECTION & $2,239,445$ & 24.2 & $25,676,463$ & 1,342 & 1,343 & 10,995 & 56,426 & 69 \\
\hline & $Z_{\text {IPPER }}^{e}$ & $1,110,244$ & 12.5 & $8,650,411$ & 1,032 & 1,261 & 10,440 & 51,261 & 119 \\
\hline & SCALER & $1,062,721$ & 11.8 & $12,096,236$ & 1,120 & 1,197 & 10,639 & 52,063 & 357 \\
\hline & BATON (Unity) & 738,878 & 8.4 & $6,905,176$ & 842 & 1,145 & 10,304 & 49,864 & 450 \\
\hline \multirow{5}{*}{ galleon } & CI & $61,536,682$ & 224.6 & $774,840,941$ & 5,063 & 7,046 & 31,137 & 195,026 & 571 \\
\hline & COLleCtion & $21,484,100$ & 80.9 & $338,974,351$ & 3,647 & 6,071 & 30,038 & 164,764 & 1,012 \\
\hline & ZIPPER $^{e}$ & $25,881,909$ & 97.8 & $330,526,330$ & 3,923 & 5,903 & 29,786 & 165,085 & 583 \\
\hline & SCALER & $28,084,055$ & 105.2 & $383,443,213$ & 3,739 & 6,175 & 30,194 & 167,957 & 4,697 \\
\hline & BATON (Unity) & $7,831,380$ & 31.5 & $110,490,509$ & 3,023 & 5,458 & 28,138 & 139,979 & 533 \\
\hline \multirow{5}{*}{ jedit } & CI & $16,874,067$ & 98.0 & $224,287,546$ & 3,382 & 4,749 & 21,006 & 118,426 & 104 \\
\hline & Collection & $3,895,227$ & 23.1 & $66,401,696$ & 2,516 & 4,152 & 20,562 & 99,574 & 79 \\
\hline & $\mathrm{ZIPPER}^{e}$ & $3,588,763$ & 21.4 & $59,605,295$ & 2,304 & 4,065 & 20,418 & 98,290 & 100 \\
\hline & SCALER & $3,583,465$ & 21.3 & $61,720,584$ & 2,377 & 3,990 & 20,499 & 97,999 & 1,580 \\
\hline & BATON (Unity) & $3,140,272$ & 18.8 & $52,291,827$ & 2,098 & 3,895 & 20,340 & 96,872 & 2,028 \\
\hline \multirow{5}{*}{ soot } & CI & $110,901,529$ & 365.2 & $2,006,757,243$ & 16,570 & 16,532 & 32,459 & 415,476 & 1,013 \\
\hline & COLlECTION & $31,431,433$ & 105.1 & $744,789,961$ & 10,296 & 14,765 & 31,952 & 367,708 & 1,221 \\
\hline & $\mathrm{ZIPPER}^{e}$ & $33,832,881$ & 113.1 & $859,999,654$ & 10,673 & 14,666 & 31,965 & 326,092 & 797 \\
\hline & SCALER & $34,952,536$ & 116.8 & $817,587,505$ & 10,549 & 14,822 & 31,982 & 374,877 & 1,257 \\
\hline & BATON (Unity) & $24,080,734$ & 80.7 & $579,686,802$ & 9,483 & 14,499 & 31,878 & 308,306 & 574 \\
\hline \multirow{5}{*}{ gruntspud } & CI & $23,261,792$ & 112.6 & $301,389,233$ & 3,583 & 5,703 & 24,887 & 148,874 & 171 \\
\hline & Collection & $8,099,569$ & 40.1 & $103,258,215$ & 2,636 & 4,820 & 24,210 & 120,835 & 137 \\
\hline & ZIPPER $^{e}$ & $7,670,883$ & 38.2 & $104,660,677$ & 2,549 & 4,739 & 24,117 & 120,316 & 288 \\
\hline & SCALER & $7,577,272$ & 37.6 & $100,355,028$ & 2,479 & 4,699 & 24,207 & 120,177 & 2,761 \\
\hline & BATON (Unity) & $6,275,349$ & 31.4 & $81,988,438$ & 2,096 & 4,572 & 23,992 & 116,842 & 3,254 \\
\hline \multirow{5}{*}{ heritrix } & CI & $19,576,304$ & 120.3 & $208,124,549$ & 2,514 & 3,264 & 18,820 & 117,551 & 198 \\
\hline & Collection & $6,268,412$ & 41.0 & $71,227,109$ & 1,628 & 2,553 & 17,549 & 94,501 & 217 \\
\hline & $\mathrm{ZIPPER}^{e}$ & $7,179,587$ & 47.2 & $78,727,889$ & 1,734 & 2,501 & 17,419 & 96,633 & 348 \\
\hline & SCALER & $7,106,210$ & 46.7 & $80,018,315$ & 1,637 & 2,461 & 17,461 & 94,785 & 2,601 \\
\hline & BATON (Unity) & $2,546,396$ & 17.7 & $20,679,819$ & 1,200 & 2,182 & 16,428 & 82,301 & 847 \\
\hline \multirow{5}{*}{ pmd } & CI & $7,713,272$ & 57.8 & $86,502,490$ & 2,948 & 4,183 & 15,254 & 104,457 & 67 \\
\hline & Collection & $2,765,814$ & 21.1 & $28,677,101$ & 2,237 & 3,684 & 14,967 & 94,750 & 74 \\
\hline & $Z_{\text {IPPER }}{ }^{e}$ & $2,760,540$ & 21.1 & $29,809,145$ & 2,153 & 3,634 & 14,908 & 93,516 & 386 \\
\hline & SCALER & $2,410,883$ & 18.4 & $28,460,705$ & 2,176 & 3,536 & 14,895 & 92,775 & 697 \\
\hline & BATON (Unity) & $2,141,253$ & 16.5 & $24,976,066$ & 1,956 & 3,463 & 14,803 & 91,762 & 3,602 \\
\hline \multirow{5}{*}{ jython } & CI & $16,581,997$ & 157.7 & $183,658,141$ & 2,234 & 2,778 & 12,718 & 114,856 & 108 \\
\hline & Collection & $13,281,462$ & 131.5 & $148,452,466$ & 1,901 & 2,572 & 12,205 & 107,903 & 152 \\
\hline & $Z_{\text {IPPER }}^{e}$ & $12,948,335$ & 129.8 & $142,634,911$ & 1,781 & 2,486 & 12,026 & 107,113 & 134 \\
\hline & SCALER & $13,016,241$ & 129.1 & $134,214,889$ & 1,852 & 2,500 & 12,167 & 107,410 & 459 \\
\hline & BATON (Unity) & $12,687,718$ & 127.3 & $129,984,727$ & 1,719 & 2,438 & 11,997 & 106,645 & 773 \\
\hline \multirow{5}{*}{ jasperreports } & CI & $73,783,904$ & 276.9 & $586,687,036$ & 5,305 & 7,060 & 33,019 & 187,173 & 577 \\
\hline & COLlection & $14,935,335$ & 59.9 & $151,406,154$ & 3,501 & 5,544 & 30,774 & 152,250 & 627 \\
\hline & $Z_{\text {IPPER }}^{e}$ & $32,834,048$ & 126.5 & $258,484,882$ & 4,202 & 6,083 & 32,044 & 167,340 & 1,043 \\
\hline & SCALER & $19,025,792$ & 75.9 & $176,489,911$ & 3,615 & 5,577 & 30,904 & 154,745 & 1,551 \\
\hline & BATON (Unity) & $6,453,254$ & 26.5 & $74,908,878$ & 2,994 & 5,267 & 30,008 & 142,256 & 834 \\
\hline \multirow{5}{*}{ eclipse } & CI & $24,841,670$ & 123.4 & $261,199,033$ & 4,190 & 9,197 & 20,862 & 161,222 & 136 \\
\hline & COLlECTION & $11,366,316$ & 57.4 & $70,520,064$ & 3,266 & 8,657 & 20,523 & 146,770 & 122 \\
\hline & ZIPPER $^{e}$ & $11,848,840$ & 59.8 & $90,933,963$ & 3,223 & 8,599 & 20,499 & 148,037 & 3,052 \\
\hline & SCALER & $11,117,708$ & 56.4 & $73,208,882$ & 3,211 & 8,486 & 20,374 & 145,953 & 680 \\
\hline & BATON (Unity) & $9,817,684$ & 50.4 & $62,570,596$ & 2,834 & 8,352 & 20,142 & 143,503 & 5,965 \\
\hline \multirow{5}{*}{ briss } & CI & $34,335,976$ & 136.6 & $370,495,823$ & 4,904 & 6,297 & 26,582 & 176,785 & 233 \\
\hline & Collection & $9,454,391$ & 38.7 & $101,712,404$ & 3,416 & 5,373 & 25,706 & 153,414 & 151 \\
\hline & $\mathrm{ZIPPER}^{e}$ & $8,202,040$ & 33.7 & $85,183,405$ & 3,158 & 5,306 & 25,537 & 151,550 & 217 \\
\hline & SCALER & $9,294,471$ & 38.1 & $101,195,279$ & 3,428 & 5,323 & 25,652 & 152,761 & 1,265 \\
\hline & BATON (Unity) & $7,653,188$ & 31.5 & $79,503,155$ & 2,955 & 5,147 & 25,473 & 150,749 & 1,726 \\
\hline \multirow{5}{*}{ columba } & CI & $86,528,734$ & 336.2 & $745,538,811$ & 4,889 & 6,669 & 31,476 & 195,930 & 771 \\
\hline & COLLECTION & $8,837,513$ & 41.8 & $109,500,746$ & 2,842 & 4,774 & 25,709 & 122,187 & 218 \\
\hline & $\mathrm{ZIPPER}^{e}$ & $21,510,036$ & 100.4 & $264,213,761$ & 3,444 & 5,213 & 26,051 & 140,637 & 837 \\
\hline & SCALER & $9,986,400$ & 47.2 & $122,951,398$ & 2,897 & 4,799 & 25,729 & 123,330 & 295 \\
\hline & BATON (Unity) & $6,658,750$ & 32.1 & $85,365,064$ & 2,393 & 4,522 & 25,157 & 118,803 & 358 \\
\hline
\end{tabular}

Proc. ACM Program. Lang., Vol. 5, No. OOPSLA, Article 147. Publication date: October 2021. 


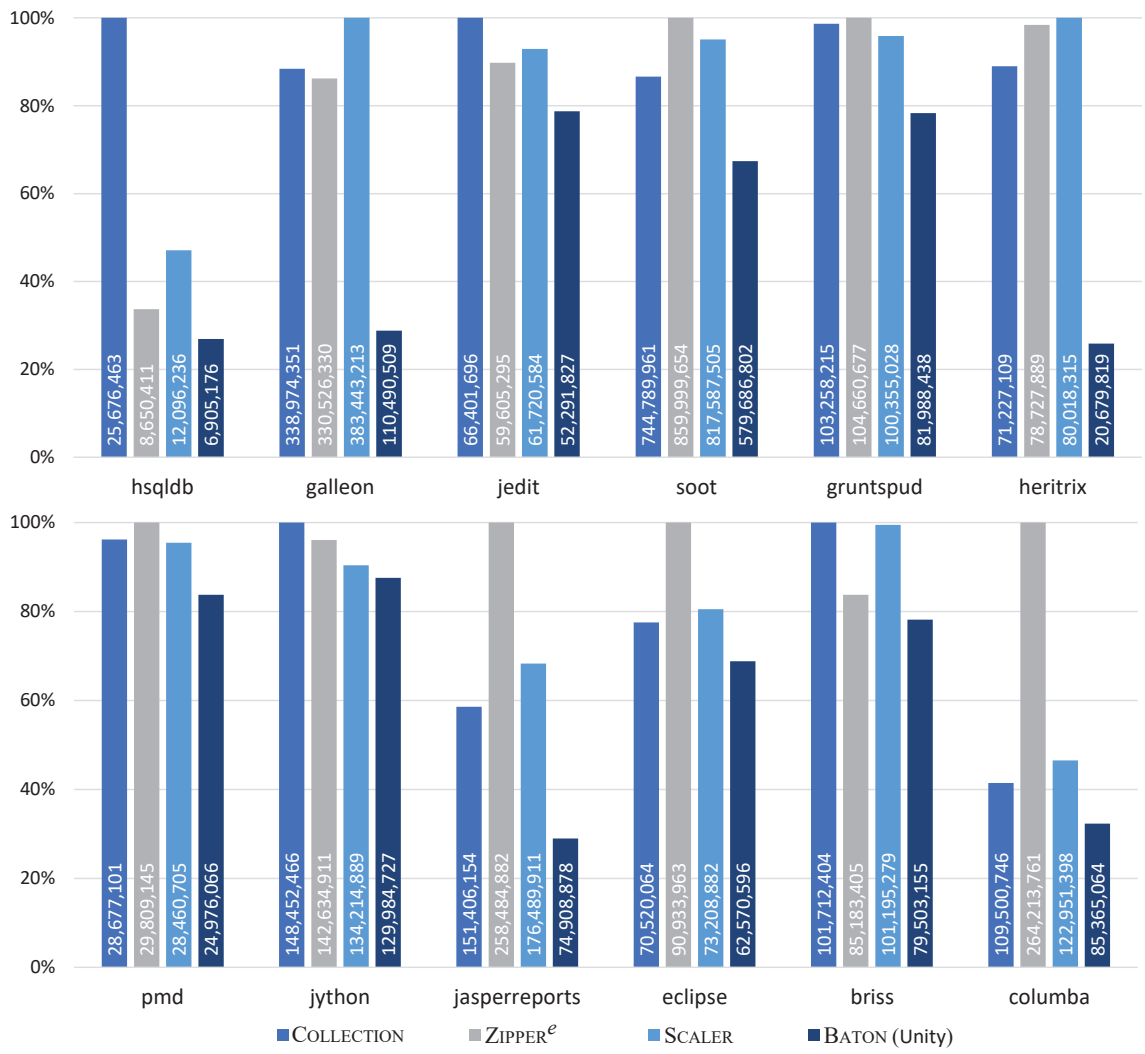

Fig. 7. May-alias variable pairs (Aliases), normalized to highest. BATON (Uni ty) consistently finds a significant proportion of previously-reported alias pairs to be spurious.

Scalability. Collection, Zipper ${ }^{e}$ and Scaler can analyze all 13 programs within time budget, demonstrating their extremely good scalability. As for BATON (Unity), even though it chooses the most precise configuration for all methods selected by all approaches in BATON, it is still able to scale for 12 out of 13 hard-to-analyze programs as shown in Table 1, validating the insights of Section 3.2. The only exception is h2, which will be handled by BATON (Relay) as shown in Section 6.2. Although our focus is precision, it is worth pointing out that BATON (Unity) is always more precise but not always slower. For galleon, heritrix, soot, jasperreports and columba, BATON (Unity) is faster than at least one of the state-of-the-art systems. For galleon and soot, BATON (Unity) is even faster than all other analyses including CI! Such surprising performance advantages stem from the significant precision improvements of BATON (Unity).

\subsection{RQ2. Precision and Scalability of BATON (Relay).}

We evaluate how BATON (Relay), as the second punch of BATON, performs in practice when BATON (Unity) is not scalable. With default settings of ZIPPER ${ }^{e}$ and ScAlER, BATON (Unity) can scale for all programs except for h2 (Section 6.1). However, to thoroughly evaluate BATON (Relay), we need to examine more such cases. ZIPPER ${ }^{e}$ and SCALER are tunable, i.e., they both provide options to tune the analysis for different precision and efficiency trade-offs. Specifically, ZIPPER ${ }^{e}$ can be configured by a percentage value, $\mathrm{PV}$, which corresponds to the threshold for identifying scalability-threat 
Table 2. Precision and performance metrics for context-insensitive CI and selective context-sensitive pointer analyses guided by reconfigured ZIPPER ${ }^{e}$, SCALER, and each pass of BATON (Relay). For all numbers, lower is better.

\begin{tabular}{|c|c|c|c|c|c|c|c|c|c|}
\hline Program & Analysis & VarPts & AvgPts & Aliases & FCasts & PCalls & RMtds & CEdges & Time (s) \\
\hline \multirow{6}{*}{ eclipse } & CI & $24,841,670$ & 123.4 & $261,199,033$ & 4,190 & 9,197 & 20,862 & 161,222 & 136 \\
\hline & $Z_{\text {IPPER }}^{e}$ & $11,848,840$ & 59.8 & $90,933,963$ & 3,223 & 8,599 & 20,499 & 148,037 & 3,052 \\
\hline & SCALER & $10,975,564$ & 55.7 & $72,760,368$ & 3,170 & 8,448 & 20,352 & 145,412 & 1,396 \\
\hline & BATON-p1 (Relay-o1) & $11,366,316$ & 57.4 & $70,520,064$ & 3,266 & 8,657 & 20,523 & 146,770 & 122 \\
\hline & BATON-p2 (Relay-o1) & $10,484,964$ & 53.2 & $65,384,654$ & 3,003 & 8,534 & 20,426 & 145,849 & 3,592 \\
\hline & BATON-p3 (Relay-o1) & $9,865,071$ & 50.7 & $62,549,586$ & 2,823 & 8,335 & 20,127 & 143,046 & 3,171 \\
\hline \multirow{6}{*}{ briss } & $\mathrm{CI}$ & $34,335,976$ & 136.6 & $370,495,823$ & 4,904 & 6,297 & 26,582 & 176,785 & 233 \\
\hline & $Z_{\text {IPPER }}^{e}$ & $5,966,496$ & 24.6 & $80,559,736$ & 3,016 & 5,209 & 25,440 & 150,826 & 1,899 \\
\hline & SCALER & $8,830,552$ & 36.4 & $97,300,729$ & 3,298 & 5,190 & 25,541 & 151,224 & 4,706 \\
\hline & BATON-p1 (Relay-o1) & $9,454,391$ & 38.7 & $101,712,404$ & 3,416 & 5,373 & 25,706 & 153,414 & 151 \\
\hline & BATON-p2 (Relay-o1) & $5,279,719$ & 21.8 & $77,196,053$ & 2,918 & 5,147 & 25,432 & 150,621 & 2,976 \\
\hline & BATON-p3 (Relay-o1) & $5,178,884$ & 21.4 & $75,371,504$ & 2,807 & 5,028 & 25,378 & 149,864 & 6,205 \\
\hline \multirow{6}{*}{ pmd } & CI & $7,713,272$ & 57.8 & $86,502,490$ & 2,948 & 4,183 & 15,254 & 104,457 & 67 \\
\hline & $Z_{\text {IPPER }}^{e}$ & $2,760,540$ & 21.1 & $29,809,145$ & 2,153 & 3,634 & 14,908 & 93,516 & 386 \\
\hline & SCALER & $2,282,726$ & 17.5 & $26,945,008$ & 2,080 & 3,498 & 14,839 & 92,439 & 2,230 \\
\hline & BATON-p1 (Relay-o1) & $2,765,814$ & 21.1 & $28,677,101$ & 2,237 & 3,684 & 14,967 & 94,750 & 74 \\
\hline & BATON-p2 (Relay-o1) & $2,558,733$ & 19.6 & $26,892,448$ & 2,072 & 3,616 & 14,891 & 93,164 & 1,827 \\
\hline & BATON-p3 (Relay-o1) & $2,142,179$ & 16.5 & $24,966,489$ & 1,958 & 3,462 & 14,802 & 91,764 & 6,164 \\
\hline \multirow{6}{*}{ jedit } & CI & $16,874,067$ & 98.0 & $224,287,546$ & 3,382 & 4,749 & 21,006 & 118,426 & 104 \\
\hline & $Z_{\text {IPPER }}^{e}$ & $2,771,120$ & 16.6 & $51,199,984$ & 2,148 & 3,937 & 20,350 & 96,946 & 3,055 \\
\hline & SCALER & $3,059,304$ & 18.3 & $56,958,326$ & 2,228 & 3,909 & 20,386 & 97,154 & 1,520 \\
\hline & BATON-p1 (Relay-o1) & $3,895,227$ & 23.1 & $66,401,696$ & 2,516 & 4,152 & 20,562 & 99,574 & 81 \\
\hline & BATON-p2 (Relay-o1) & $2,627,200$ & 15.7 & $49,384,180$ & 2,113 & 3,930 & 20,346 & 96,763 & 3,887 \\
\hline & BATON-p3 (Relay-o1) & $2,575,332$ & 15.4 & $48,120,051$ & 2,022 & 3,845 & 20,327 & 96,463 & 4,347 \\
\hline \multirow{6}{*}{ h2 } & CI & $3,566,057$ & 30.1 & $66,745,220$ & 1,866 & 3,946 & 14,192 & 95,541 & 49 \\
\hline & $Z_{\text {IPPER }}^{e}$ & $1,571,052$ & 13.5 & $26,790,820$ & 1,418 & 3,646 & 13,903 & 89,292 & 254 \\
\hline & SCALER & $1,368,866$ & 11.8 & $26,113,035$ & 1,373 & 3,605 & 13,850 & 88,268 & 1,832 \\
\hline & BATON-p1 (Relay-o1) & $1,647,387$ & 14.1 & $26,677,699$ & 1,523 & 3,654 & 13,935 & 89,361 & 63 \\
\hline & BATON-p2 (Relay-o1) & $1,480,200$ & 12.7 & $22,915,240$ & 1,384 & 3,632 & 13,867 & 88,959 & 2,284 \\
\hline & BATON-p3 (Relay-o2) & $1,238,765$ & 10.7 & $22,264,106$ & 1,303 & 3,593 & 13,821 & 88,027 & 2,004 \\
\hline \multirow{6}{*}{ gruntspud } & CI & $23,261,792$ & 112.5 & $301,389,233$ & 3,583 & 5,703 & 24,887 & 148,874 & 171 \\
\hline & $Z_{\text {IPPER }}^{e}$ & $5,601,249$ & 28.0 & $79,231,338$ & 2,232 & 4,629 & 24,010 & 116,972 & 1,370 \\
\hline & SCALER & $6,928,500$ & 34.4 & $99,005,787$ & 2,470 & 4,671 & 24,166 & 119,136 & 4,546 \\
\hline & BATON-p1 (Relay-o1) & $8,099,569$ & 40.1 & $103,258,215$ & 2,636 & 4,820 & 24,210 & 120,835 & 137 \\
\hline & BATON-p2 (Relay-o2) & $5,525,251$ & 27.6 & $78,100,575$ & 2,199 & 4,625 & 24,001 & 116,892 & 1,456 \\
\hline & BATON-p3 (Relay-o2) & $5,356,169$ & 26.8 & $76,524,253$ & 2,113 & 4,541 & 23,973 & 116,558 & 3,962 \\
\hline
\end{tabular}

methods, and SCALER is parameterized by a number called total scalability threshold (TST), which corresponds to a bound on the overall points-to size. For both analyses, using higher thresholds (PV and TST) would generally improve precision but reduce efficiency. Thus, we change their settings in BATON by increasing PV and TST, to make them more precise, and accordingly, more expensive, until BATON (Unity) no longer scales but each individual selective approach can still terminate within the time budget for all the programs in Table 1.

As a result, Table 2 shows six programs that fulfill our experimental requirements. As the baseline, CI is still included. The results of Collection are the same as the ones of BATON-p1 (Relay-o1) (since BAton (Relay) runs Collection in its first pass as described in Section 5), thus we use the latter to represent Collection. For each program, we list the analysis results of the reconfigured ZIPPER $^{e}$, SCALER, and the three passes (Section 5) of the accordingly-reconfigured BATON (Relay).

Precision. Theorem 4.6 proves that the last pass of Relay is more precise than any individual selective approach in the framework, and Table 2 validates this in practice: BATON (Relay) obtains better precision than ZIPPER ${ }^{e}$ and SCALER (and also COLLECTION) for all precision metrics for all programs. Figure 8 shows graphically the precision improvement for the Aliases metric. 


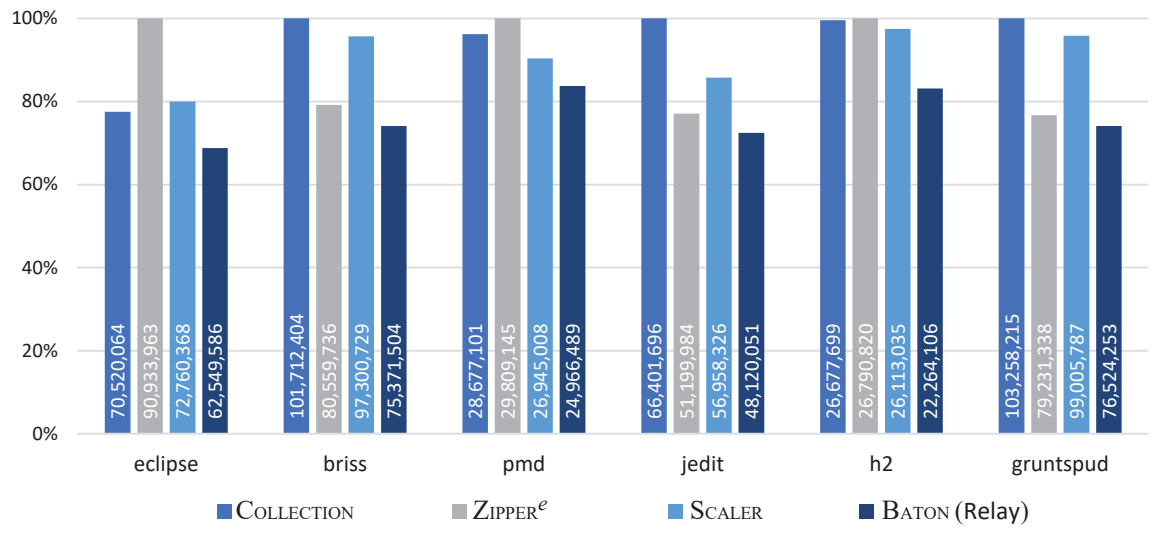

Fig. 8. May-alias variable pairs (Aliases), normalized to highest. BAton (Relay) consistently improves over the best past algorithm. Importantly, the best algorithm varies per benchmark. On average, BATON (Relay) improves the precision of COLLECTION, ZIPPER ${ }^{e}$, and SCALER by $20 \%, 13 \%$ and $16 \%$ respectively.

We can also observe from Table 2 that BAton (Relay) improves the precision in a stable, cumulative way, pass after pass (BATON-p1, -p2 and -p3). For 4 out of 6 programs, the precision achieved by the second pass of BATON (Relay), i.e., BATON-p2, already outperforms the other analyses.

Scalability. For all six programs in Table 2, for which the reconfigured BATON (Unity) fails to scale, BAton (Relay) is scalable, by dividing the scalability burden of BATON (Unity) into different passes. The beneficial effects of on-the-fly filtering, based on the results of the previous pass, can be seen in the experiment. For instance, one can observe that, for gruntspud, BATON-p3 (Relay-o2) spends 3,962s when the corresponding SCALER analysis requires 4,546s. This is entirely due to the precision transmitted from passes 1 and 2, which can help filter many spurious object flows during the analysis of pass 3, making the analysis reach fixed point more quickly. Although the purpose of filtering is to improve precision, and not scalability, when the time saved by the improved precision exceeds the time cost of filtering itself, such results arise.

Pass Ordering. We conduct further experiments to examine whether the pass ordering of Relay affects precision and scalability. The present Relay runs Collection, ZIPPER ${ }^{e}$ and SCALER, in this order, for each pass. In the extra experiments, we have two new orderings: (1) Collection, ScAler and ZipPeR ${ }^{e}$ and (2) SCALER, ZIPPER ${ }^{e}$ and COLLECTION. We run Relay for all the programs in Table 2 under these two orderings. For scalability, as expected, Relay-02 is still able to scale for all the programs, keeping its scalability promise. For Relay-01, the ScALER-related pass fails to scale for briss under the new orderings: the context-sensitivity configuration in Relay-01 for SCALER is relatively expensive in terms of analysis efficiency, but when running ZIPPER ${ }^{e}$ before SCALER, as in the original ordering, the former helps prune away many spurious objects, which makes the latter more scalable by reaching fixed point faster. This instance suggests that a pass ordering with relatively fast and precise analyses placed first can lead to a more scalable Relay-01, thus avoiding the fallback to the less-precise Relay-02. Again, the pass ordering does not affect the scalability capacity of Relay-02, as explained in Section 4.4, and validated above.

For precision, as expected, Relay outperforms the other analyses in all precision metrics under both new orderings. (For the aforementioned briss case, note the fallback to Relay-02.) This further validates the Relay precision guarantee. Regarding the final precision produced by Relay under the original and the two new orderings, although one may perform better than the others for 
different programs, the precision difference among them is very minor: less than $0.1 \%$ on average. Therefore, although the pass ordering may affect the final precision of Relay, as explained in Section 3.3, the experimental results show that such impact is very minor in practice. (The precision guarantee of Relay proved in Theorem 4.6 remains unaffected.)

Other Choices for Precision Improvements. When BATON (Unity) fails to scale, one may wonder whether some other schemes are better choices than Relay for improving precision. This may include: (1) downgrading the context-sensitivity variants for the overlapped methods reported by ZIPPER $^{e}$, SCALER and COLLECTION (e.g., choose the faster 2 type rather than $20 b j$ if both are reported), (2) simply intersecting the points-to results produced by ZIPPER ${ }^{e}$, SCALER and Collection, and (3) further tuning ZIPPER ${ }^{e}$ or SCALER for better precision by increasing their provided thresholds.

We conduct further experiments and find that none of the above schemes is better than BATON (Relay) for improving precision. For (1), this scheme still fails to scale for jedit, and for the remaining $5 \times 7=35$ precision metrics, BATON (Relay) outperforms it in almost all (33 out of 35) cases. For (2), BAton (Relay) achieves better precision for all 42 metrics. For (3), when increasing the current threshold of $\mathrm{ZIPPER}^{e}$ (used in Table 2) by only $1 \%$, (e.g., from $16 \%$ to $17 \%$ for gruntspud), $\mathrm{ZIPPER}^{e}$ is already not scalable for several programs; when increasing the threshold of SCALER from current $60 \mathrm{M}$ to a high value $100 \mathrm{M}$, ScALER generally becomes significantly slower (e.g., 6,856s for briss), but BATON (Relay) (with the current 60M) still outperforms it in precision for virtually all (40 out of 42) metrics. Most importantly, no matter how precise an input selective approach becomes, BATON (Relay) can always leverage it for better precision.

\section{RELATED WORK}

To our knowledge, Unity-Relay is the first technique that systematically exploits and leverages multiple selective context-sensitivity approaches for yielding high precision for Java pointer analysis. As experimental results show, Unity-Relay is able to produce a pointer analysis with a precision that is well beyond existing approaches for hard-to-analyze programs. Although the ideas of Unity and Relay have not been applied to pointer analysis before, for Relay, the high-level idea of its precision filtering is similar to the reduced product approach in abstract interpretation [Cousot and Cousot 1979]: different domains (e.g., an integer interval domain and a parity domain) can be used to improve the precision of another in one analysis; however, in Relay, there is only one domain (the mapping from pointers to heap objects) and the precision filtering process is divided into multiple analyses (passes). The high-level idea of staged analyses in Relay has also been adopted in static analysis, e.g., in staged verification [Fink et al. 2008], faster verifiers run in early stages which reduce the workload for later, more precise, stages; however, in Relay, neither the time cost nor the precision of the analysis in each stage are comparable. Relay is also different in how it utilizes the results from a previous stage to incorporate with the analysis in a later stage. (Such handling also differs from staged optimization [Philipose et al. 2002].) Thus, although Relay shares some similarities with the high-level ideas of previous work, technical specifics are different.

We have discussed Zipper ${ }^{e}$ [Li et al. 2020], ScAler [Li et al. 2018b] and Collection [WALA 2018] in Section 5. Below we review other related work about selective context-sensitive pointer analysis.

Oh et al. [2014] present a principled approach to resolving given queries for C programs by selective context sensitivity. In particular, the approach uses a pre-analysis to estimate the impact of context sensitivity on the precision of the subsequent main analysis. In Oh et al. [2015], this approach is extended to include selective flow sensitivity by following the same principle to demonstrate its generality. 
Smaragdakis et al. [2014] introduce introspective analysis, which relies on several manuallyselected metrics (e.g., the maximum field points-to set per object) to define heuristics for determining which methods are supposed to be analyzed context-sensitively. Similar parameterized heuristics are also leveraged by Hassanshahi et al [Hassanshahi et al. 2017] to decide whether object sensitivity should be applied to certain methods.

Like SCALER [Li et al. 2018b], context elements may also vary for different methods in the work of Wei and Ryder [2015] and Thakur and Nandivada [2020]. Differently, in Wei and Ryder [2015], the relationship between context-sensitivity variants and methods is obtained by a machine-learning approach and domain knowledge. In the work of Thakur and Nandivada [2020], object sensitivity is further added to the methods that have been assigned call-site sensitivity, in a special form of context abstraction proposed earlier by Thakur and Nandivada [2019].

Data-driven approaches [Jeon et al. 2019; Jeong et al. 2017] assign to each method an appropriate context length by learning heuristics based on various program elements (e.g., some Java keywords or specific statements) expressed in a disjunctive form. Although the learning phase is relatively heavy, it helps in making good precision and efficiency trade-offs for pointer analysis. However, as well-known weaknesses, machine-learning approaches may behave unpredictably for new inputs, and their learned results, although useful, are usually difficult to explain. Still, this does not prevent the ability of Unity-Relay to take advantage of learned results (based on opaque insights from machine-learning algorithms) to gain good precision, just as it can for any other individual selective approach.

$\mathrm{Lu}$ and Xue [2019] present a scheme to selectively apply context sensitivity on the granularity of variables (rather than methods) based on CFL-reachability [Reps 1997]. The general idea of Uni ty-Relay also works for this granularity by considering which portion of a program should be analyzed by what context-sensitivity variants at the variable level (see the circles in Figures 2 and 3 as selected variables).

It is worth pointing out that Unity-Relay is not in competition with the above approaches, but instead complements them by taking advantages of their insights (through consuming their outputs) to yield more precise results.

When we understand selective context sensitivity more generally as "applying context sensitivity discriminately, in order to save analysis cost" it becomes similar to ideas also explored in demand/client-driven pointer analyses [Guyer and Lin 2003; Liang and Naik 2011; Liu et al. 2019; Späth et al. 2019; Späth et al. 2016; Sridharan and Bodík 2006; Sridharan et al. 2005; Sui and Xue 2016; Wang et al. 2017; Zhang et al. 2014]: these reduce the analysis cost by computing only the results which are necessary to answer specific queries at given program locations.

\section{CONCLUSIONS}

Pointer analysis is hard to scale with good precision for large and complex Java programs. This challenge needs to be urgently addressed, as such programs dominate real practice. To address the problem, we present the simple and practical Unity-Relay framework for systematically exploiting a set, $S$, of selective context-sensitivity approaches, to produce highly-precise pointer analysis results for hard-to-analyze Java programs. Its first punch, Unity, fully unleashes the precision potential of all approaches in $S$ with good scalability. When Unity fails, the second punch, Relay, can be confidently relied upon to reap precision in a stable and accumulative way. Unity-Relay has soundness and precision guarantees relative to the individual selective approaches it combines. Furthermore, if each approach in $S$ is scalable, users can expect that Unity-Relay is also able to scale by its last defender of scalability, Relay-02: a technique guaranteed to (at worst) match the scalability profile of individual approaches in $S$. We validate these properties in practice via extensive experiments in Section 6. 
The Unity-Relay proof-of-concept tool, BATON, has been demonstrated to be very effective: BATON achieves the best precision for all precision metrics and clients for all evaluated programs. It is the first time that these levels of precision are obtained for these hard-to-analyze programs.

BATON is merely one instantiation of the Unity-Relay framework. In the future, we expect more instantiations to unleash the power of more effective selective context-sensitivity approaches (including ones not yet foreseen).

\section{ACKNOWLEDGMENTS}

We thank the anonymous reviewers for their helpful comments. This work was supported in part by Key-Area Research \& Development Program of Guangdong Province (Grant \#2020B010164003), Leading-edge Technology Program of Jiangsu Natural Science Foundation (Grant \#BK20202001), and National Natural Science Foundation of China (Grants \#61932021, \#62025202, \#62002157), and by the Hellenic Foundation for Research and Innovation (project DEAN-BLOCK). The authors would also like to thank the support from the Collaborative Innovation Center of Novel Software Technology and Industrialization, Jiangsu, China.

\section{REFERENCES}

Steven Arzt, Siegfried Rasthofer, Christian Fritz, Eric Bodden, Alexandre Bartel, Jacques Klein, Yves Le Traon, Damien Octeau, and Patrick D. McDaniel. 2014. FlowDroid: precise context, flow, field, object-sensitive and lifecycle-aware taint analysis for Android apps. In ACM SIGPLAN Conference on Programming Language Design and Implementation, PLDI '14, Edinburgh, United Kingdom - June 09 - 11, 2014, Michael F. P. O’Boyle and Keshav Pingali (Eds.). ACM, 259-269. https://doi.org/10.1145/2594291.2594299

Stephen M. Blackburn, Robin Garner, Chris Hoffmann, Asjad M. Khan, Kathryn S. McKinley, Rotem Bentzur, Amer Diwan, Daniel Feinberg, Daniel Frampton, Samuel Z. Guyer, Martin Hirzel, Antony L. Hosking, Maria Jump, Han Bok Lee, J. Eliot B. Moss, Aashish Phansalkar, Darko Stefanovic, Thomas VanDrunen, Daniel von Dincklage, and Ben Wiedermann. 2006. The DaCapo benchmarks: Java benchmarking development and analysis. In Proceedings of the 21th Annual ACM SIGPLAN Conference on Object-Oriented Programming, Systems, Languages, and Applications, OOPSLA 2006, October 22-26, 2006, Portland, Oregon, USA, Peri L. Tarr and William R. Cook (Eds.). ACM, 169-190. https://doi.org/10.1145/1167473.1167488

Eric Bodden, Andreas Sewe, Jan Sinschek, Hela Oueslati, and Mira Mezini. 2011. Taming reflection: Aiding static analysis in the presence of reflection and custom class loaders. In Proceedings of the 33rd International Conference on Software Engineering, ICSE 2011, Waikiki, Honolulu, HI, USA, May 21-28, 2011. 241-250. https://doi.org/10.1145/1985793.1985827

Martin Bravenboer and Yannis Smaragdakis. 2009. Strictly declarative specification of sophisticated points-to analyses. In Proceedings of the 24th Annual ACM SIGPLAN Conference on Object-Oriented Programming, Systems, Languages, and Applications, OOPSLA 2009, October 25-29, 2009, Orlando, Florida, USA, Shail Arora and Gary T. Leavens (Eds.). ACM, 243-262. https://doi.org/10.1145/1640089.1640108

Satish Chandra, Stephen J. Fink, and Manu Sridharan. 2009. Snugglebug: a powerful approach to weakest preconditions. In Proceedings of the 2009 ACM SIGPLAN Conference on Programming Language Design and Implementation, PLDI 2009, Dublin, Ireland, fune 15-21, 2009, Michael Hind and Amer Diwan (Eds.). ACM, 363-374. https://doi.org/10.1145/1542476.1542517

Maria Christakis and Christian Bird. 2016. What developers want and need from program analysis: an empirical study. In Proceedings of the 31st IEEE/ACM International Conference on Automated Software Engineering, ASE 2016, Singapore, September 3-7, 2016, David Lo, Sven Apel, and Sarfraz Khurshid (Eds.). ACM, 332-343. https://doi.org/10.1145/2970276. 2970347

Patrick Cousot and Radhia Cousot. 1979. Systematic Design of Program Analysis Frameworks. In Proceedings of the 6th ACM SIGACT-SIGPLAN Symposium on Principles of Programming Languages (San Antonio, Texas) (POPL '79). Association for Computing Machinery, New York, NY, USA, 269-282. https://doi.org/10.1145/567752.567778

Pratik Fegade and Christian Wimmer. 2020. Scalable Pointer Analysis of Data Structures Using Semantic Models. In Proceedings of the 29th International Conference on Compiler Construction (San Diego, CA, USA) (CC 2020). Association for Computing Machinery, New York, NY, USA, 39-50. https://doi.org/10.1145/3377555.3377885

Yu Feng, Xinyu Wang, Isil Dillig, and Thomas Dillig. 2015. Bottom-Up Context-Sensitive Pointer Analysis for Java. In Programming Languages and Systems, Xinyu Feng and Sungwoo Park (Eds.). Springer International Publishing, Cham, 465-484.

Stephen J. Fink, Eran Yahav, Nurit Dor, G. Ramalingam, and Emmanuel Geay. 2008. Effective Typestate Verification in the Presence of Aliasing. ACM Trans. Softw. Eng. Methodol. 17, 2, Article 9 (May 2008), 34 pages. https://doi.org/10.1145/ 1348250.1348255

Proc. ACM Program. Lang., Vol. 5, No. OOPSLA, Article 147. Publication date: October 2021. 
Samuel Z. Guyer and Calvin Lin. 2003. Client-Driven Pointer Analysis. In Proceedings of the 10th International Conference on Static Analysis (San Diego, CA, USA) (SAS'03). Springer-Verlag, Berlin, Heidelberg, 214-236.

Behnaz Hassanshahi, Raghavendra Kagalavadi Ramesh, Padmanabhan Krishnan, Bernhard Scholz, and Yi Lu. 2017. An efficient tunable selective points-to analysis for large codebases. In Proceedings of the 6th ACM SIGPLAN International Workshop on State Of the Art in Program Analysis, SOAP@PLDI 2017, Barcelona, Spain, June 18, 2017, Karim Ali and Cristina Cifuentes (Eds.). ACM, 13-18. https://doi.org/10.1145/3088515.3088519

Minseok Jeon, Sehun Jeong, Sungdeok Cha, and Hakjoo Oh. 2019. A Machine-Learning Algorithm with Disjunctive Model for Data-Driven Program Analysis. ACM Trans. Program. Lang. Syst. 41, 2 (2019), 13:1-13:41. https://doi.org/10.1145/3293607

Minseok Jeon, Sehun Jeong, and Hakjoo Oh. 2018. Precise and Scalable Points-to Analysis via Data-driven Context Tunneling. Proc. ACM Program. Lang. 2, OOPSLA, Article 140 (Oct. 2018), 29 pages. https://doi.org/10.1145/3276510

Sehun Jeong, Minseok Jeon, Sung Deok Cha, and Hakjoo Oh. 2017. Data-driven context-sensitivity for points-to analysis. PACMPL 1, OOPSLA (2017), 100:1-100:28. https://doi.org/10.1145/3133924

Vini Kanvar and Uday P. Khedker. 2016. Heap Abstractions for Static Analysis. ACM Comput. Surv. 49, 2, Article 29 (June 2016), 47 pages. https://doi.org/10.1145/2931098

George Kastrinis and Yannis Smaragdakis. 2013. Hybrid context-sensitivity for points-to analysis. In ACM SIGPLAN Conference on Programming Language Design and Implementation, PLDI '13, Seattle, WA, USA, June 16-19, 2013, HansJuergen Boehm and Cormac Flanagan (Eds.). ACM, 423-434. https://doi.org/10.1145/2491956.2462191

Ondřej Lhoták and Laurie Hendren. 2008. Evaluating the Benefits of Context-Sensitive Points-to Analysis Using a BDD-Based Implementation. ACM Trans. Softw. Eng. Methodol. 18, 1, Article 3 (Oct. 2008), 53 pages. https://doi.org/10.1145/1391984. 1391987

Ondrej Lhoták and Laurie J. Hendren. 2006. Context-Sensitive Points-to Analysis: Is It Worth It?. In Compiler Construction, 15th International Conference, CC 2006, Held as Part of the foint European Conferences on Theory and Practice of Software, ETAPS 2006, Vienna, Austria, March 30-31, 2006, Proceedings (Lecture Notes in Computer Science, Vol. 3923), Alan Mycroft and Andreas Zeller (Eds.). Springer, 47-64. https://doi.org/10.1007/11688839_5

Yue Li, Tian Tan, Anders Møller, and Yannis Smaragdakis. 2018a. Precision-guided Context Sensitivity for Pointer Analysis. Proc. ACM Program. Lang. 2, OOPSLA, Article 141 (Oct. 2018), 29 pages. https://doi.org/10.1145/3276511

Yue Li, Tian Tan, Anders Møller, and Yannis Smaragdakis. 2018b. Scalability-First Pointer Analysis with Self-Tuning ContextSensitivity. In Proc. 12th joint meeting of the European Software Engineering Conference and the ACM SIGSOFT Symposium on the Foundations of Software Engineering (ESEC/FSE). ACM, 129-140. https://doi.org/10.1145/3236024.3236041

Yue Li, Tian Tan, Anders Møller, and Yannis Smaragdakis. 2020. A Principled Approach to Selective Context Sensitivity for Pointer Analysis. ACM Trans. Program. Lang. Syst. 42, 2, Article 10 (May 2020), 40 pages. https://doi.org/10.1145/3381915

Yue Li, Tian Tan, Yifei Zhang, and Jingling Xue. 2016. Program Tailoring: Slicing by Sequential Criteria. In 30th European Conference on Object-Oriented Programming, ECOOP 2016, fuly 18-22, 2016, Rome, Italy (LIPIcs, Vol. 56), Shriram Krishnamurthi and Benjamin S. Lerner (Eds.). Schloss Dagstuhl - Leibniz-Zentrum fuer Informatik, 15:1-15:27. https://doi.org/10.4230/LIPIcs.ECOOP.2016.15

Percy Liang and Mayur Naik. 2011. Scaling Abstraction Refinement via Pruning. SIGPLAN Not. 46, 6 (June 2011), 590-601. https://doi.org/10.1145/1993316.1993567

Bozhen Liu, Jeff Huang, and Lawrence Rauchwerger. 2019. Rethinking Incremental and Parallel Pointer Analysis. ACM Trans. Program. Lang. Syst. 41, 1, Article 6 (March 2019), 31 pages. https://doi.org/10.1145/3293606

Benjamin Livshits and Monica S. Lam. 2005. Finding Security Vulnerabilities in Java Applications with Static Analysis. In Proceedings of the 14th USENIX Security Symposium, Baltimore, MD, USA, fuly 31 - August 5, 2005, Patrick D. McDaniel (Ed.). USENIX Association.

Jingbo Lu and Jingling Xue. 2019. Precision-Preserving yet Fast Object-Sensitive Pointer Analysis with Partial Context Sensitivity. Proc. ACM Program. Lang. 3, OOPSLA, Article 148 (Oct. 2019), 29 pages. https://doi.org/10.1145/3360574

Ana Milanova, Atanas Rountev, and Barbara G. Ryder. 2002. Parameterized object sensitivity for points-to and side-effect analyses for Java. In Proceedings of the International Symposium on Software Testing and Analysis, ISSTA 2002, Roma, Italy, fuly 22-24, 2002, Phyllis G. Frankl (Ed.). ACM, 1-11. https://doi.org/10.1145/566172.566174

Ana Milanova, Atanas Rountev, and Barbara G. Ryder. 2005. Parameterized object sensitivity for points-to analysis for Java. ACM Trans. Softw. Eng. Methodol. 14, 1 (2005), 1-41. https://doi.org/10.1145/1044834.1044835

Myungho Lee Minseok Jeon and Hakjoo Oh. 2020. Learning Graph-based Heuristics for Pointer Analysis without Handcrafting Application-Specific Features. Proc. ACM Program. Lang. OOPSLA (2020).

Mayur Naik, Alex Aiken, and aley. 2006. Effective static race detection for Java. In Proceedings of the ACM SIGPLAN 2006 Conference on Programming Language Design and Implementation, Ottawa, Ontario, Canada, June 11-14, 2006, Michael I. Schwartzbach and Thomas Ball (Eds.). ACM, 308-319. https://doi.org/10.1145/1133981.1134018

Hakjoo Oh, Wonchan Lee, Kihong Heo, Hongseok Yang, and Kwangkeun Yi. 2014. Selective Context-sensitivity Guided by Impact Pre-analysis. In Proceedings of the 35th ACM SIGPLAN Conference on Programming Language Design and Implementation (Edinburgh, United Kingdom) (PLDI '14). ACM, New York, NY, USA, 475-484. https://doi.org/10.1145/ 
2594291.2594318

Hakjoo Oh, Wonchan Lee, Kihong Heo, Hongseok Yang, and Kwangkeun Yi. 2015. Selective X-Sensitive Analysis Guided by Impact Pre-Analysis. ACM Trans. Program. Lang. Syst. 38, 2, Article 6 (Dec. 2015), 45 pages. https://doi.org/10.1145/ 2821504

Matthai Philipose, Craig Chambers, and Susan J. Eggers. 2002. Towards Automatic Construction of Staged Compilers. In Proceedings of the 29th ACM SIGPLAN-SIGACT Symposium on Principles of Programming Languages (Portland, Oregon) (POPL '02). Association for Computing Machinery, New York, NY, USA, 113-125. https://doi.org/10.1145/503272.503284

Michael Pradel, Ciera Jaspan, Jonathan Aldrich, and Thomas R. Gross. 2012. Statically checking API protocol conformance with mined multi-object specifications. In 34th International Conference on Software Engineering, ICSE 2012, June 2-9, 2012, Zurich, Switzerland, Martin Glinz, Gail C. Murphy, and Mauro Pezzè (Eds.). IEEE Computer Society, 925-935. https://doi.org/10.1109/ICSE.2012.6227127

Thomas Reps. 1997. Program Analysis via Graph Reachability. In Proceedings of the 1997 International Symposium on Logic Programming (Port Washington, New York, USA) (ILPS '97). MIT Press, Cambridge, MA, USA, 5-19.

Yannis Smaragdakis and George Balatsouras. 2015. Pointer Analysis. Foundations and Trends in Programming Languages 2 , 1 (2015), 1-69. https://doi.org/10.1561/2500000014

Yannis Smaragdakis, Martin Bravenboer, and Ondrej Lhoták. 2011. Pick your contexts well: understanding object-sensitivity. In Proceedings of the 38th ACM SIGPLAN-SIGACT Symposium on Principles of Programming Languages, POPL 2011, Austin, TX, USA, fanuary 26-28, 2011, Thomas Ball and Mooly Sagiv (Eds.). ACM, 17-30. https://doi.org/10.1145/1926385 1926390

Yannis Smaragdakis, George Kastrinis, and George Balatsouras. 2014. Introspective analysis: context-sensitivity, across the board. In ACM SIGPLAN Conference on Programming Language Design and Implementation, PLDI '14, Edinburgh, United Kingdom - fune 09 - 11, 2014, Michael F. P. O'Boyle and Keshav Pingali (Eds.). ACM, 485-495. https://doi.org/10.1145/ 2594291.2594320

Johannes Späth, Karim Ali, and Eric Bodden. 2019. Context-, Flow-, and Field-Sensitive Data-Flow Analysis Using Synchronized Pushdown Systems. Proc. ACM Program. Lang. 3, POPL, Article 48 (Jan. 2019), 29 pages. https: //doi.org/10.1145/3290361

Johannes Späth, Lisa Nguyen Quang Do, Karim Ali, and Eric Bodden. 2016. Boomerang: Demand-Driven Flow- and Context-Sensitive Pointer Analysis for Java. In 30th European Conference on Object-Oriented Programming (ECOOP 2016) (Leibniz International Proceedings in Informatics (LIPIcs), Vol. 56), Shriram Krishnamurthi and Benjamin S. Lerner (Eds.) Schloss Dagstuhl-Leibniz-Zentrum fuer Informatik, Dagstuhl, Germany, 22:1-22:26. https://doi.org/10.4230/LIPIcs. ECOOP.2016.22

Manu Sridharan and Rastislav Bodík. 2006. Refinement-based context-sensitive points-to analysis for Java. In Proceedings of the ACM SIGPLAN 2006 Conference on Programming Language Design and Implementation, Ottawa, Ontario, Canada, June 11-14, 2006, Michael I. Schwartzbach and Thomas Ball (Eds.). ACM, 387-400. https://doi.org/10.1145/1133981.1134027

Manu Sridharan, Satish Chandra, Julian Dolby, Stephen J. Fink, and Eran Yahav. 2013. Alias Analysis for Object-Oriented Programs. In Aliasing in Object-Oriented Programming. Types, Analysis and Verification, Dave Clarke, James Noble, and Tobias Wrigstad (Eds.). Lecture Notes in Computer Science, Vol. 7850. Springer, 196-232. https://doi.org/10.1007/978-3642-36946-9_8

Manu Sridharan, Stephen J. Fink, and Rastislav Bodík. 2007. Thin slicing. In Proceedings of the ACM SIGPLAN 2007 Conference on Programming Language Design and Implementation, San Diego, California, USA, June 10-13, 2007, Jeanne Ferrante and Kathryn S. McKinley (Eds.). ACM, 112-122. https://doi.org/10.1145/1250734.1250748

Manu Sridharan, Denis Gopan, Lexin Shan, and Rastislav Bodík. 2005. Demand-driven points-to analysis for Java. In Proceedings of the 20th Annual ACM SIGPLAN Conference on Object-Oriented Programming, Systems, Languages, and Applications, OOPSLA 2005, October 16-20, 2005, San Diego, CA, USA, Ralph E. Johnson and Richard P. Gabriel (Eds.). ACM, 59-76. https://doi.org/10.1145/1094811.1094817

Yulei Sui and Jingling Xue. 2016. On-demand Strong Update Analysis via Value-flow Refinement. In Proceedings of the 2016 24th ACM SIGSOFT International Symposium on Foundations of Software Engineering (Seattle, WA, USA) (FSE 2016). ACM, New York, NY, USA, 460-473. https://doi.org/10.1145/2950290.2950296

Tian Tan, Yue Li, Xiaoxing Ma, Chang Xu, and Yannis Smaragdakis. 2021. Making Pointer Analysis More Precise by Unleashing the Power of Selective Context Sensitivity (Artifact). https://doi.org/10.5281/zenodo.5491895

Tian Tan, Yue Li, and Jingling Xue. 2016. Making k-Object-Sensitive Pointer Analysis More Precise with Still k-Limiting. In Static Analysis - 23rd International Symposium, SAS 2016, Edinburgh, UK, September 8-10, 2016, Proceedings (Lecture Notes in Computer Science, Vol. 9837), Xavier Rival (Ed.). Springer, 489-510. https://doi.org/10.1007/978-3-662-53413-7_24

Tian Tan, Yue Li, and Jingling Xue. 2017. Efficient and precise points-to analysis: modeling the heap by merging equivalent automata. In Proceedings of the 38th ACM SIGPLAN Conference on Programming Language Design and Implementation, PLDI 2017, Barcelona, Spain, fune 18-23, 2017, Albert Cohen and Martin T. Vechev (Eds.). ACM, 278-291. https://doi.org/ 10.1145/3062341.3062360 
Manas Thakur and V. Krishna Nandivada. 2019. Compare Less, Defer More: Scaling Value-contexts Based Whole-program Heap Analyses. In Proceedings of the 28th International Conference on Compiler Construction (Washington, DC, USA) (CC 2019). ACM, New York, NY, USA, 135-146. https://doi.org/10.1145/3302516.3307359

Manas Thakur and V. Krishna Nandivada. 2020. Mix Your Contexts Well: Opportunities Unleashed by Recent Advances in Scaling Context-Sensitivity (CC 2020). Association for Computing Machinery, New York, NY, USA, 27-38. https: //doi.org/10.1145/3377555.3377902

Rei Thiessen and Ondřej Lhoták. 2017. Context Transformations for Pointer Analysis. In Proceedings of the 38th ACM SIGPLAN Conference on Programming Language Design and Implementation (Barcelona, Spain) (PLDI 2017). ACM, New York, NY, USA, 263-277. https://doi.org/10.1145/3062341.3062359

WALA. 2018. Watson Libraries for Analysis. http://wala.sf.net.

Kai Wang, Aftab Hussain, Zhiqiang Zuo, Guoqing Xu, and Ardalan Amiri Sani. 2017. Graspan: A Single-machine Disk-based Graph System for Interprocedural Static Analyses of Large-scale Systems Code. In Proceedings of the Twenty-Second International Conference on Architectural Support for Programming Languages and Operating Systems (Xi'an, China) (ASPLOS '17). ACM, New York, NY, USA, 389-404. https://doi.org/10.1145/3037697.3037744

Shiyi Wei and Barbara G. Ryder. 2015. Adaptive Context-sensitive Analysis for JavaScript. In 29th European Conference on Object-Oriented Programming, ECOOP 2015, fuly 5-10, 2015, Prague, Czech Republic (LIPIcs, Vol. 37), John Tang Boyland (Ed.). Schloss Dagstuhl - Leibniz-Zentrum fuer Informatik, 712-734. https://doi.org/10.4230/LIPIcs.ECOOP.2015.712

John Whaley and Monica S. Lam. 2004. Cloning-Based Context-Sensitive Pointer Alias Analysis Using Binary Decision Diagrams. SIGPLAN Not. 39, 6 (June 2004), 131-144. https://doi.org/10.1145/996893.996859

Guoqing Xu and Atanas Rountev. 2008. Merging Equivalent Contexts for Scalable Heap-cloning-based Context-sensitive Points-to Analysis. In Proceedings of the 2008 International Symposium on Software Testing and Analysis (Seattle, WA, USA) (ISSTA '08). ACM, New York, NY, USA, 225-236. https://doi.org/10.1145/1390630.1390658

Xin Zhang, Ravi Mangal, Radu Grigore, Mayur Naik, and Hongseok Yang. 2014. On Abstraction Refinement for Program Analyses in Datalog. In Proceedings of the 35th ACM SIGPLAN Conference on Programming Language Design and Implementation (Edinburgh, United Kingdom) (PLDI '14). Association for Computing Machinery, New York, NY, USA, 239-248. https://doi.org/10.1145/2594291.2594327 